\title{
Relative Velocity of Inertial Particles in Turbulent Flows
}

\author{
LIUBIN PA N $\mathbf{N}^{1,2} \dagger$ AND PAOLO PADOA N $\mathbf{N}^{1,3}$ \\ ${ }^{1}$ Department of Physics, University of California, San Diego, CASS/UCSD 0424, 9500 Gilman \\ Drive, La Jolla, CA 92093-0424 \\ ${ }^{2}$ School of Earth and Space Exploration, Arizona State University, P.O. Box 871404, Tempe, \\ AZ 85287-1404 \\ ${ }^{3}$ ICREA \& ICC, University of Barcelona, Marti i Franquès 1, E-08028 Barcelona, Spain
}

(Received ?? and in revised form ??)

We present a model for the relative velocity of inertial particles in turbulent flows that provides new physical insight into this problem. Our general formulation shows that the relative velocity has contributions from two terms, referred to as the generalized acceleration and generalized shear terms, because they reduce to the well known acceleration and shear terms in the Saffman-Turner limit. The generalized shear term represents particles' memory of the flow velocity difference along their trajectories and depends on the inertial particle pair dispersion backward in time. The importance of this backward dispersion in determining the particle relative velocity is emphasized. We find that our model with a two-phase separation behavior, an early ballistic phase and a later tracer-like phase, as found by recent simulations for the forward (in time) dispersion of inertial particle pairs, gives good fits to the measured relative speeds from simulations at low Reynolds numbers. In the monodisperse case with identical particles, the generalized acceleration term vanishes and the relative velocity is determined by the generalized shear term. At large Reynolds numbers, our model gives a $S t^{1 / 2}$ dependence of the relative velocity on the Stokes number $S t$ in the inertial range for both the ballistic behavior and the Richardson separation law. This leads to the same inertial-range scaling for the two-phase separation that well fits the simulation results. Our calculations for the bidisperse case show that, with the friction timescale of one particle fixed, the relative speed as a function of the other particle's friction time has a dip when the two timescales are similar. This indicates that similar-size particles tend to have stronger velocity correlation than different ones. We find that the primary contribution at the dip, i.e., for similar particles, is from the generalized shear term, while the generalized acceleration term is dominant for particles of very different sizes. Future numerical studies are motivated to check the accuracy of the assumptions made in our model and to investigate the backward-in-time dispersion of inertial particle pairs in turbulent flows.

Key Words: Particle/fluid flows; Turbulent flows

\section{Introduction}

The dynamics of inertial particles suspended in turbulent flows is of both theoretical and practical importance. Its applications range from industrial processes, e.g., turbulent spray combustion, aerosols and raindrop formation in terrestrial clouds, and dust grain

$\dagger$ Email address for correspondence: liubin.pan@asu.edu 
dynamics in astrophysical environments such as interstellar media, protoplanetary disks, and planetary atmospheres. Particle collisions in a turbulent flow are of particular interest because they determine the growth of the particles by coagulation. The estimate of the collision rate requires the understanding of two interesting phenomena regarding inertial particles in turbulence, the preferential concentration and the turbulence-induced relative velocity. The latter is the focus of the present work.

Our primary motivations for this study is its application to theoretical modeling of planetesimal formation in protoplanetory disks. Planetesimals are objects of kilometer size that can further grow into fully-fledged planets. The formation of these objects is believed to start from the growth of dust grains of micrometer size by collisional coagulation (e.g., Weidenschilling 1980). Particles involved in this process have an extensive size range, from micrometer to kilometer, which corresponds to a range of friction timescale that covers all the scales (from the dissipation range, the inertial range to the outer scales) in the turbulence in protoplanetary disks. Therefore, a detailed understanding of particle collision velocities for a whole range of particle sizes induced by turbulence motions is crucial to investigating particle growth in these disks. Dust grain collisions in protoplanetary disks do not always lead to coagulation. The grains become less sticky as the size increases. If the relative speed is large, the collision between two large particles may cause them to fragment or to simply bounce off each other (e.g., Blum \& Wurm 2008). To judge the outcome of collisions between large particles thus requires an accurate prediction for the collision speed.

Besides turbulence, there are other effects that can induce relative velocities between particles. For example, gravity gives rise to differential settling for particles of different sizes, which can have important contribution to the relative speed between these particles. In astrophysical environments, radiation pressure and the coupling with magnetic fields (through electric charge on the grain surface) can also play an important role. In the present study, we will neglect these effects, and concentrate on the turbulence-induced relative velocity. We aim at a physical understanding of relative velocities induced by turbulent motions alone, which is clearly a crucial step toward an accurate model for the particle collisions in the presence of both turbulence and the other complexities. The model presented here could be extended to take the other effects into account. Although our work is motivated by the problem of dust grain collisions in astrophysical environments, it has applications in other contexts such as droplet formation in cloud physics (e.g., Saffman \& Turner 1956).

The relative velocity of two nearby particles induced by turbulent motions has been extensively studied. Saffman \& Turner (1956) considered particles with small inertia in the limit that the friction time, $\tau_{p}$, of both particles is much smaller than the Kolmogorov timescale, $\tau_{\eta}$. This limit is also expressed as $S t \ll 1$, where the Stokes number, $S t$, is defined as the ratio of $\tau_{p}$ to $\tau_{\eta}$. In this limit, the particle velocity at a given point can be approximately obtained from the (1st-order) Taylor expansion of the particle momentum equation (eq. (2.1) below). For two particles with a separation, $r$, much smaller than the Kolmogorov scale, $\eta$, Saffman \& Turner (1956) derived a result for the average radial relative velocity, $\left\langle\left|w_{r}\right|\right\rangle$. In the absence of gravity, there are two terms that contribute to the relative speed, a shear term and an acceleration term (see Ayala et al. 2008),

$$
\left\langle\left|w_{r}\right|\right\rangle=\sqrt{\frac{2}{\pi}}\left(\frac{1}{15} \frac{\bar{\epsilon}}{\nu} r^{2}+\left(\tau_{p 2}-\tau_{p 1}\right)^{2} a^{2}\right)^{1 / 2}
$$

where $\bar{\epsilon}$ and $\nu$ are, respectively, the average dissipation rate and the kinematic viscosity of the turbulent flow, $\tau_{p 1}$ and $\tau_{p 2}$ are the friction timescales of the two particles, and $a$ 
is the rms acceleration, i.e., $a^{2}=\left\langle\left(\frac{D u}{D t}\right)^{2}\right\rangle$. The factor $\sqrt{2 / \pi}$ is from the conversion of the radial relative velocity variance, $\left\langle w_{r}^{2}\right\rangle$, to $\left\langle\left|w_{r}\right|\right\rangle$ assuming a Gaussian distribution for $w_{r}$. Note that the shear term is from the longitudinal structure function, $S_{l l}(r)$, of the flow, which is given by $\frac{1}{15} \frac{\bar{\epsilon}}{\nu} r^{2}$ for $r \lesssim \eta$. In the Saffman-Turner (S-T) limit, the particle velocity follows the local flow velocity very closely, thus the velocity of the two particles at a distance $r<\eta$ is highly correlated because of the strong flow velocity correlation across a small distance.

The opposite limit is that of heavy particles with friction timescales much larger than the Lagrangian correlation timescale, $T_{L}$. In this limit, the velocities of two particles coming together are essentially uncorrelated. This is because particles with $\tau_{p} \gg T_{L}$ have long-time memory, and their current velocities have substantial contributions from the flow velocities on their trajectories in the past. These flow velocities at early times are likely to be uncorrelated because the particles were far away from each other. The relative velocity in this limit is thus determined by the sum of the velocity variances of the two particles,

$$
\left\langle\left|w_{r}\right|\right\rangle=\sqrt{\frac{2}{\pi}}\left[\left(v^{\prime(1)}\right)^{2}+\left(v^{\prime(2)}\right)^{2}\right]^{1 / 2}
$$

where $v^{\prime(1)}$ and $v^{\prime(2)}$ denote the particle rms velocities. In the limit $\tau_{p} \gg T_{L}$, they are given by (e.g., Abrahamson 1975),

$$
\left(v^{\prime(1)}\right)^{2} \simeq u^{\prime 2} \frac{T_{L}}{\tau_{p 1}} ; \quad\left(v^{\prime(2)}\right)^{2} \simeq u^{\prime 2} \frac{T_{L}}{\tau_{p 2}}
$$

where $u^{\prime}$ is the rms of the flow velocity fluctuations. In the derivation of eq. (1.3), the temporal correlation of the flow velocity on a particle's trajectory is approximated by the Lagrangian correlation for tracer particles. Discussions on the validity of this assumption will be given in $\S 2.2$.

The problem of the relative velocity of inertial particles in these two extreme limits is physically clear, and the results given above are easy to understand and are expected to be generally robust. On the other hand, for particles with intermediate inertia, $\tau_{\eta} \lesssim \tau_{p} \lesssim T_{L}$, the problem is more complicated and is less well understood. The velocities of two nearby particles with intermediate $\tau_{p}$ are partially correlated and the degree of correlation, intermediate between the two limits, is not easy to evaluate. We will point out that a very important factor in determining this correlation is the distance between the trajectories of the two particles as a function of time before they come close to each other. To our knowledge, this point has not been clearly recognized or explicitly emphasized in the literature. We will show how the separation of two nearby inertial particles backward in time affects the relative velocity between particles with intermediate inertia, $\tau_{\eta} \lesssim \tau_{p} \lesssim T_{L}$.

A successful theory for particles of all sizes needs to correctly predict and explain the behavior of the relative velocity between particles with intermediate friction time, as well as recover the results in the two extreme limits. An example of particular theoretical interest is that of identical particles, referred to as the monodisperse case. In the S-T limit $\left(\tau_{p} \ll \tau_{\eta}\right)$, the acceleration term in eq. (1.1) vanishes for the monodisperse case and the relative velocity does not depend on the friction time. It is constant at a given distance, $r$, and increases linearly with $r$. In the opposite limit with $\tau_{p} \gg T_{L}$, the relative velocity decreases with the friction time as $\tau_{p}^{-1 / 2}$ or $S t^{-1 / 2}$, according to eqs. (1.2) and (1.3). The question of how $\left\langle\left|w_{r}\right|\right\rangle$ scales with $\tau_{p}$ for $\tau_{p}$ in a range corresponding to the inertial range of the turbulent flow, and how it connects with the two extreme limits has 
not been systematically studied or fully understood. This is one of the primary goals of the present paper. We find that the dispersion of particles backward in time is crucial to answer this question.

The existing models have very different predictions for the relative speed in the inertial range for the monodisperse case (Volk et al. 1980; Williams \& Crane 1983; Yuu 1984; Kruis \& Kusters 1997; Zaichik et al. 2003, 2006; Avala et al. 2008). A detailed discussion of the qualitative differences between these models and their problems will be given in $\S 4$. An important reason for the problems in most of the previous models is that they did not clearly recognize or carefully consider the effect of the particle pair separation backward in time (except for the differential model by Zaichik et al. (2003, 2006) to be discussed below, which we think has the particle backward separation implicitly included). The role of this backward separation will be revealed along the formulation of our model.

In the previous studies that cover a whole range of Stokes numbers, the differential model by Zaichik and collaborators (Zaichik \& Alipchenkov 2003; Zaichik et al. 2003, 2006) is perhaps the most complete one, as it examines the effect of preferential clustering and the relative speed simultaneously. We will refer to this model as Zaichik et al.'s model or the model by Zaichik et al. Assuming Gaussian statistics for the flow velocity, the model first sets up an equation for the joint probability distribution function (pdf) of the particle separation and the relative velocity. Deriving the first 3 moment equations of the pdf equation and closing these moment equations by a quasi-normal approximation, Zaichik et al. were able to obtain a set of partial differential equations for the particle density correlation function (usually referred to as the radial distribution function) and the particle velocity structure functions. The solution of the differential equations reproduces the two extreme limits discussed above and predicts that the relative velocity of identical particles with intermediate inertia is proportional to $\tau_{p}^{1 / 2}$ or, equivalently, $S t^{1 / 2}$. The validity of this prediction remains to be confirmed by high-resolution simulations. Despite the elegant mathematical formulation and good agreement with results of direct numerical simulations of turbulence with low Reynolds numbers, the model lacks physical transparency in its approximations, especially the quasi-normal assumption. One of the goals of our model is to elucidate the physics behind the inertial range scaling of the relative velocity.

Falkovich et al. (2002) found that an effect, named the sling effect, has a significant contribution to the relative speed (see also Wilkinson \& Mehlig 2005; Wilkinson et al. 2006; Falkovich \& Pumir 2007). The physical picture of the effect is that, at regions with large negative velocity gradients, faster moving particles can catch up the slower ones from behind (Falkovich et al. 2002), leading to trajectory crossing of the particles (Bec et al. (2005), also see Fig. 1 in Falkovich \& Pumir (2007) for an illustration). This results in a larger relative speed than the S-T prediction for small particles. Falkovich \& Pumir (2007) showed that the effect starts to be important for $S t \gtrsim 0.2$ and gives a relative speed several time larger than eq (1.1) for $S t$ between 0.2 and 1 . We will point out a common element shared by the sling effect and our model: the contribution to the relative speed from the particles' memory of the flow velocity difference in the past.

The paper is organized as follows. We present the formulation of our model in $\S 2$ (a general formulation in $\S 2.1$, and basic assumptions and approximations in $\S 2.2$ and $\S 2.3$ ). The results for identical particles (monodisperse) and different particles (bidisperse) are given in $\S 3.1$ and $\S 3.2$, respectively. In $\S 4$, we discuss previous models and compare them with our model. Conclusions are given in $\S 5$. 


\section{The Model}

\subsection{The General Formulation}

The velocity, $\boldsymbol{v}(t)$, of a particle with friction time, $\tau_{p}$, can be obtained by integrating the momentum equation,

$$
\frac{d \boldsymbol{v}}{d t}=\frac{\boldsymbol{u}(\boldsymbol{X}(t), t)-\boldsymbol{v}}{\tau_{p}}
$$

where $\boldsymbol{u}(\boldsymbol{x}, t)$ denotes the flow velocity field and $\boldsymbol{X}(t)$ is the position of the particle at time $t$. Clearly, $\boldsymbol{u}(\boldsymbol{X}(t), t)$ is the flow velocity at the positions of the particle along its trajectory (we will refer to it also as the flow velocity "seen" by the particle). The particle trajectory is given by,

$$
\boldsymbol{X}(t)=\boldsymbol{X}_{0}+\int_{t_{0}}^{t} \boldsymbol{v}\left(t^{\prime}\right) d t^{\prime}
$$

where $\boldsymbol{X}_{0}$ is the particle position at a given time $t_{0}$.

Equation (2.1) has a formal solution,

$$
\boldsymbol{v}(t)=\boldsymbol{v}_{0} \exp \left(-\frac{t-t_{0}}{\tau_{p}}\right)+\frac{1}{\tau_{p}} \int_{t_{0}}^{t} \boldsymbol{u}(\boldsymbol{X}(\tau), \tau) \exp \left(-\frac{t-\tau}{\tau_{p}}\right) d \tau
$$

where $\boldsymbol{v}_{0}$ is the particle velocity at $t_{0}$.

We are interested in deriving the relative velocity between two particles at a distance $\boldsymbol{r}$ at a given time $t$. We label the two particles by superscripts "(1)" and "(2)". For example, their velocities at $t$ are denoted as $\boldsymbol{v}^{(1)}(t)$ and $\boldsymbol{v}^{(2)}(t)$, respectively. To evaluate the average relative speed, we will calculate the velocity structure tensor, $S_{\mathrm{p} i j}$, of the two particles,

$$
S_{\mathrm{p} i j}(\boldsymbol{r}, t)=\left\langle\left(v_{i}^{(1)}-v_{i}^{(2)}\right)\left(v_{j}^{(1)}-v_{j}^{(2)}\right)\right\rangle
$$

where $\langle\cdots\rangle$ denotes the ensemble average. The particle velocities can be solved by integrating equation (2.3) and the trajectories of the two particles are subject to a constraint,

$$
\boldsymbol{X}^{(1)}(t)-\boldsymbol{X}^{(2)}(t)=\boldsymbol{r} .
$$

which means that two particles happen to be separated by $\boldsymbol{r}$ at $t$. We will particularly consider small values of $r$ (below $\eta$ ) because we are interested in the collision speed, which is essentially the relative velocity of the two particles over a distance of the particle size. From the structure tensor $S_{\mathrm{p} i j}$, we will obtain the longitudinal structure function $S_{\mathrm{p} l l}$, which, by definition, is the radial relative velocity variance, $\left\langle w_{r}^{2}\right\rangle$. Although only small $r$ will be considered in the paper, our model can predict the structure function at all separations. By a comparison with their results, our model may provide an explanation for the inertial particle structure functions found in Bec et al. (2009b).

The particle structure tensor can be written as,

$$
S_{\mathrm{p} i j}=\left\langle v_{i}^{(1)} v_{j}^{(1)}\right\rangle-\left\langle v_{i}^{(1)} v_{j}^{(2)}\right\rangle-\left\langle v_{i}^{(2)} v_{j}^{(1)}\right\rangle+\left\langle v_{i}^{(2)} v_{j}^{(2)}\right\rangle .
$$

Note that the cross terms correspond to the particle velocity correlations discussed in $\S 1$, where it was argued that a careful treatment of such correlations is essential for modeling the relative velocity of particles with intermediate inertia.

To calculate the structure tensor, we insert eq. (2.3) for the particle velocities into eq. (2.6). For simplicity in notations, we will set the time when the particle relative speed is measured (i.e., $t$ in eq. (2.4)) to be zero, and assume it is far from the initial time (since we are interested in the relative velocity for a statistically stationary state). This allows us to set $t_{0}$ in eq. (2.3) to $-\infty$. 
We analyze the four terms in eq. (2.6) one by one. The first term on the r.h.s. corresponds to the velocity variance of particle (1). For this term, only the velocity of particle (1) is involved and we have,

$$
\left\langle v_{i}^{(1)} v_{j}^{(1)}\right\rangle=\int_{-\infty}^{0} \frac{d \tau}{\tau_{p 1}} \int_{-\infty}^{0} \frac{d \tau^{\prime}}{\tau_{p 1}}\left\langle u_{i}^{(1)}(\tau) u_{j}^{(1)}\left(\tau^{\prime}\right)\right\rangle \exp \left(\frac{\tau}{\tau_{p 1}}\right) \exp \left(\frac{\tau^{\prime}}{\tau_{p 1}}\right)
$$

where $u_{i}^{(1)}(t)=u_{i}\left(\boldsymbol{X}^{(1)}(t), t\right)$ denotes the flow velocity on the trajectory of particle (1). The exponential factors here represent the memory loss of the particles. The integral limits in eq. (2.7) (see also eq. (2.9)) suggest that it is the flow velocity the particles saw in the past that is relevant in determining the particles' velocities at the current time. The relative position of the two particles back in time will be shown to play an important role in the prediction of their relative velocity. We will call $\left\langle u_{i}^{(1)}(\tau) u_{j}^{(1)}\left(\tau^{\prime}\right)\right\rangle$ in the integral the trajectory correlation tensor and denote it as $B_{\mathrm{T} i j}^{(1)}$, i.e.,

$$
B_{\mathrm{T} i j}^{(1)}\left(\tau, \tau^{\prime}\right)=\left\langle u_{i}^{(1)}(\tau) u_{j}^{(1)}\left(\tau^{\prime}\right)\right\rangle
$$

where the subscript " $\mathrm{T}$ " stands for "trajectory".

The result for the 4 th term on the rhs of eq. (2.6) is similar. One only needs to replace $\tau_{p 1}$ in eq. (2.7) by $\tau_{p 2}$, and $B_{\mathrm{T} i j}^{(1)}$ by $B_{\mathrm{T} i j}^{(2)} \equiv\left\langle u_{i}^{(2)}(\tau) u_{j}^{(2)}\left(\tau^{\prime}\right)\right\rangle$. If the two particles are identical and have the same friction time, $\left\langle v_{i}^{(1)} v_{j}^{(1)}\right\rangle$ is equal to $\left\langle v_{i}^{(2)} v_{j}^{(2)}\right\rangle$. These two terms correspond to the velocity variance of each particle and will be called the velocity variance terms.

The exact form of $B_{\mathrm{T} i j}$ as a function of the friction time is not available. In the limit of vanishing $\tau_{p}$ (i.e., passive tracers), this correlation tensor would approach the Lagrangian correlation tensor, $B_{\mathrm{L} i j}$, of the flow, which has been extensively studied (e.g., Yeung \& Pope 1989). A common approximation is to set $B_{\mathrm{T} i j}$ equal to $B_{\mathrm{L} i j}$ for particles with any $\tau_{p}$ (e.g., Zaichik \& Alipchenkov 2003; Zaichik et al. 2003, 2006; Avala et al. 2008). Physically, it corresponds to the assumption that the trajectory of any inertial particle is not far away from that of a tracer particle starting from the same initial condition. We will adopt this assumption in our calculations and its validity will be discussed in $\S 2.2$.

The cross correlation terms in eq. (2.6) can be evaluated with the same approach. The second term on the r.h.s. is given by,

$$
\left\langle v_{i}^{(1)} v_{j}^{(2)}\right\rangle=\int_{-\infty}^{0} \frac{d \tau}{\tau_{p 1}} \int_{-\infty}^{0} \frac{d \tau^{\prime}}{\tau_{p 2}}\left\langle u_{i}^{(1)}(\tau) u_{j}^{(2)}\left(\tau^{\prime}\right)\right\rangle \exp \left(\frac{\tau}{\tau_{p 1}}\right) \exp \left(\frac{\tau^{\prime}}{\tau_{p 2}}\right) .
$$

The result for the term $\left\langle v_{i}^{(2)} v_{j}^{(1)}\right\rangle$ in eq. (2.6) is similar to eq. (2.9). The sum of these two tensors can be written as,

$$
\left\langle u_{i}^{(1)}(\tau) u_{j}^{(2)}\left(\tau^{\prime}\right)\right\rangle+\left\langle u_{i}^{(2)}(\tau) u_{j}^{(1)}\left(\tau^{\prime}\right)\right\rangle=B_{\mathrm{T} i j}^{(1)}\left(\tau, \tau^{\prime}\right)+B_{\mathrm{T} i j}^{(2)}\left(\tau, \tau^{\prime}\right)-S_{\mathrm{T} i j}\left(\boldsymbol{r} ; \tau, \tau^{\prime}\right)
$$

where the tensor $S_{\text {T } i j}$ is defined as

$$
S_{\mathrm{T} i j}\left(\boldsymbol{r} ; \tau, \tau^{\prime}\right)=\left\langle\left[u_{i}^{(1)}(\tau)-u_{i}^{(2)}(\tau)\right]\left[u_{j}^{(1)}\left(\tau^{\prime}\right)-u_{j}^{(2)}\left(\tau^{\prime}\right)\right]\right\rangle .
$$

Clearly, $S_{\text {Tij }}$ is the correlation of the flow velocity difference at the positions of the particles on their trajectories at times $\tau$ and $\tau^{\prime}$. We have explicitly indicated the dependence of the tensor on the particle separation at time zero. The ensemble average on the r.h.s. 
includes an average over the probability distribution of the flow velocity difference at time zero. By analogy with $B_{\mathrm{T} i j}$, we will call $S_{\mathrm{T} i j}$ the trajectory structure tensor.

Since $S_{\text {Tij }}$ has not been directly studied, we will give an approximate estimate for it in $\S 2.3$. For example, we will relate the flow velocity difference, $\boldsymbol{u}^{(1)}(t)-\boldsymbol{u}^{(2)}(t)$, along the trajectories by the two particles, to the separation of the two particles at $t$, assuming the velocity difference scaling in the Eulerian frame applies to the velocity difference on the particles' trajectories. The uncertainty in this assumption will be discussed in $\S 2.3$. We will denote the particle separation at a given time $t$ as $\boldsymbol{\rho}(t)$, which, in our notation, is given by $\boldsymbol{X}^{(1)}(t)-\boldsymbol{X}^{(2)}(t)$. Note that $\boldsymbol{\rho}$ is a stochastic vector because of the particle dispersion by turbulent motions.

Combining eqs. (2.6), (2.7), (2.8), (2.9) and (2.10), we finally arrive at the formula for the velocity structure tensor of two particles separated by $\boldsymbol{r}$,

$$
S_{\mathrm{p} i j}(\boldsymbol{r})=\mathcal{A}_{i j}+\mathcal{D}_{i j},
$$

where

$$
\begin{aligned}
\mathcal{A}_{i j}= & \int_{-\infty}^{0} \frac{d \tau}{\tau_{p 1}} \int_{-\infty}^{0} \frac{d \tau^{\prime}}{\tau_{p 1}} B_{\mathrm{T} i j}^{(1)}\left(\tau, \tau^{\prime}\right) \exp \left(\frac{\tau}{\tau_{p 1}}\right) \exp \left(\frac{\tau^{\prime}}{\tau_{p 1}}\right) \\
& -\int_{-\infty}^{0} \frac{d \tau}{\tau_{p 1}} \int_{-\infty}^{0} \frac{d \tau^{\prime}}{\tau_{p 2}}\left(B_{\mathrm{T} i j}^{(1)}\left(\tau, \tau^{\prime}\right)+B_{\mathrm{T} i j}^{(2)}\left(\tau, \tau^{\prime}\right)\right) \exp \left(\frac{\tau}{\tau_{p 1}}\right) \exp \left(\frac{\tau^{\prime}}{\tau_{p 2}}\right) \\
& +\int_{-\infty}^{0} \frac{d \tau}{\tau_{p 2}} \int_{-\infty}^{0} \frac{d \tau^{\prime}}{\tau_{p 2}} B_{\mathrm{T} i j}^{(2)}\left(\tau, \tau^{\prime}\right) \exp \left(\frac{\tau}{\tau_{p 2}}\right) \exp \left(\frac{\tau^{\prime}}{\tau_{p 2}}\right)
\end{aligned}
$$

and

$$
\mathcal{D}_{i j}=\int_{-\infty}^{0} \frac{d \tau}{\tau_{p 1}} \int_{-\infty}^{0} \frac{d \tau^{\prime}}{\tau_{p 2}} S_{\mathrm{T} i j}\left(\boldsymbol{r} ; \tau, \tau^{\prime}\right) \exp \left(\frac{\tau}{\tau_{p 1}}\right) \exp \left(\frac{\tau^{\prime}}{\tau_{p 2}}\right) .
$$

In the trajectory structure tensor in $\mathcal{D}_{i j}$, the dependence on $\boldsymbol{r}$ is from the requirement that particle separation $\boldsymbol{\rho}$ is equal to $\boldsymbol{r}$ at time zero, i.e.,

$$
\boldsymbol{\rho}(0)=\boldsymbol{r} .
$$

The result, eq. (2.12), is written in such a way that the first term $\mathcal{A}_{i j}$ only depends on the 1-particle trajectory correlation tensor and the second term $\mathcal{D}_{i j}$ only on the 2 particle trajectory structure tensor. There are also physical reasons to split $S_{\mathrm{p} i j}$ into these two terms. First, the $\mathcal{A}_{i j}$ term vanishes for identical particles with $\tau_{p 1}=\tau_{p 2}$, and only $\mathcal{D}_{i j}$ contributes to the relative speed in the monodisperse case. On the other hand, for particles of very different sizes, $\mathcal{A}_{i j}$ dominates the contribution to the relative speed (see §3.2). Second, in the S-T limit, $\mathcal{A}_{i j}$ and $\mathcal{D}_{i j}$ reduce to the acceleration term and the shear term in eq. (1.1), respectively. Therefore, our formulation can be regarded as one that extends eq. (1.1) from the low-inertia limit to the whole range of particle sizes. We will refer to $\mathcal{A}_{i j}$ and $\mathcal{D}_{i j}$ as the generalized acceleration term and the generalized shear term, respectively.

It is straightforward to see that $\mathcal{D}_{i j}$ reduces to the shear contribution in the the S-T limit. As $\tau_{p} \rightarrow 0$, we have $1 / \tau_{p} \exp \left(\tau / \tau_{p}\right) \rightarrow \delta(\tau)$, and thus $\mathcal{D}_{i j}$ approaches the flow structure tensor $S_{i j}(r)$. As pointed out in $\S 1$, it is exactly this flow structure tensor that is responsible for the shear contribution in eq. (1.1).

In the S-T limit, $\mathcal{A}_{i j}$ can be evaluated as follows. For $\tau_{p} \rightarrow 0$, particle trajectories are close to those of tracers, so $B_{\mathrm{T} i j} \simeq B_{\mathrm{L} i j}$. For small time lag $\Delta \tau=\tau-\tau^{\prime}$ (only small time lag is of interest here because of the exponential cutoffs in the integrand), $B_{\mathrm{L} i j}(\Delta \tau) \simeq\left(u^{\prime 2}-\frac{1}{2} a^{2} \Delta \tau^{2}\right) \delta_{i j}$ where $u^{\prime}$ and $a$ are, respectively, the rms flow velocity and the rms acceleration. Using this approximation for both $B_{\mathrm{T} i j}^{(1)}$ and $B_{\mathrm{T} i j}^{(2)}$ in eq. (2.13), we 
find $\mathcal{A}_{i j}=a^{2}\left(\tau_{p 1}-\tau_{p 2}\right)^{2} \delta_{i j}$, which is exactly the same as the acceleration term in eq. (1.1).

In $\S 2.2$ and $\S 2.3 .3$ we will show that, with our modeling of $\mathcal{A}_{i j}$ and $\mathcal{D}_{i j}$, eqs. (2.12), (2.13), and (2.14) also recover the large particle limit as well.

Our formulation reflects the trajectory-crossing effect mentioned in $\S 1$. When setting $r$ to zero (or more exactly the particle size), the formulation is for two particles whose trajectories cross at time zero. Different from the model for the sling effect (Falkovich et al. 2002), our model does not specify the physical mechanism how and when the trajectories of two particles cross. Instead the process leading to trajectory-crossing is considered indirectly from the backward separation behavior of the two particles. From the following perspective, one may see a common feature shared by the sling effect and our model. The sling effect could be interpreted as a mechanism that contributes to the particle separation backward in time, and it gives a larger relative speed by increasing the contribution from the particles' memory of the flow velocity difference in the past. The latter is the point of our model. Therefore, we think that the sling effect can be accounted for in our model if the backward separation to be used in the model includes its contribution.

\subsection{Modeling $\mathcal{A}_{i j}$}

The formulation has been general so far. To proceed, we make assumptions for the trajectory correlation and structure tensors in eqs. (2.13) and (2.14). In this subsection, we evaluate the generalized acceleration term $\mathcal{A}_{i j}$.

We use the usual assumption for $B_{\mathrm{T} i j}$ that the flow velocity viewed by a particle on its trajectory is the same as that by a tracer particle (e.g., Zaichik \& Alipchenkov 2003; Zaichik et al. 2003, 2006; Ayala et al. 2008), i.e., $B_{\mathrm{T} i j}=B_{\mathrm{L} i j}$. In a statistically stationary and isotropic flow, the Lagrangian correlation tensor only depends on the time lag, and can be written as $B_{\mathrm{L} i j}(\Delta \tau)=u^{\prime 2} \delta_{i j} \Phi(\Delta \tau)$ where $\Phi$ is the normalized temporal correlation function. We adopt the bi-exponential form for $\Phi$ (see Sawford 1991; Zaichik et al. 2003, 2006),

$$
\begin{array}{r}
\Phi\left(\Delta \tau ; \tau_{T}, T_{L}\right)=\frac{1}{2 \sqrt{1-2 z^{2}}}\left[\left(1+\sqrt{1-2 z^{2}}\right) \exp \left(-\frac{2|\Delta \tau|}{\left(1+\sqrt{1-2 z^{2}}\right) T_{L}}\right)\right. \\
\left.-\left(1-\sqrt{1-2 z^{2}}\right) \exp \left(-\frac{2|\Delta \tau|}{\left(1-\sqrt{1-2 z^{2}}\right) T_{L}}\right)\right]
\end{array}
$$

where $T_{L}\left(=\int \Phi(\Delta \tau) d \Delta \tau\right)$ is the Lagrangian correlation timescale, and $z=\tau_{T} / T_{L}$ is the ratio of the Taylor micro timescale, $\tau_{T}$, to $T_{L}$. In addition to the time lag $\Delta \tau$, we have written $\Phi$ also as a function of $\tau_{T}$ and $T_{L}$ in eq. (2.16) for later convenience (see eq. $(2.23))$.

We obtain $T_{L}$ using DNS results for the ratio of $T_{L}$ to the large-eddy turnover time, $T_{E} . T_{E}$ is defined as the longitudinal integral scale, $L_{1}$, divided by the rms velocity, $u^{\prime}$. The length scale $L_{1}$ can be calculated from the relation, $\bar{\epsilon}=D u^{\prime 3} / L_{1}$, using simulation results for the dimensionless coefficient $D$. Defining a length scale $L$ as $L=u^{\prime 3} / \bar{\epsilon}$, we have $L_{1}=D L$. The large-eddy timescale is then given by $T_{E}=D u^{\prime 2} / \bar{\epsilon}$ or $T_{E}=D T_{e}$ with $T_{e}$ defined as $T_{e}=u^{\prime 2} / \bar{\epsilon}=L / u^{\prime}$. Yeung et al. (2006) found that the ratio of $T_{L}$ to $T_{E}$ is $\simeq 0.75$, and is essentially independent of the Taylor Reynolds number, $R e_{\lambda}$ (although this ratio may depend on the forcing and thus could be flow-dependent). Therefore we have $T_{L}=0.75 D u^{\prime 2} / \bar{\epsilon}$ or $T_{L}=0.75 D T_{e}$.

Numerical simulations have shown that the coefficient, $D$, is Reynolds number dependent for $R e_{\lambda} \lesssim 100$, but approaches a constant $\sim 0.4$ for $R e_{\lambda}$ larger than several hundred (e.g., Yeung et al. 2006; Ishihara et al. 2009). Therefore in the limit of large $R e_{\lambda}$, we ex- 
pect $T_{E}=0.3 u^{\prime 2} / \bar{\epsilon}$. To also account for the $R e_{\lambda}$ dependence of $D$ at small $R e_{\lambda}$, we use $D=0.4\left(1+30 / R e_{\lambda}\right)$, which is obtained from fitting the numerical results in Yeung et al. (2006). We then have,

$$
T_{L}=0.3\left(1+30 / R e_{\lambda}\right) u^{\prime 2} / \bar{\epsilon}
$$

which is very close to that adopted by Zaichik et al. $(2003,2006)$ and the empirical formula given in Sawford et al. (2008). The definition of $R e_{\lambda}$ gives $u^{\prime 2}=R e_{\lambda} / \sqrt{15} u_{\eta}^{2}$, which will be used for normalization in our calculations.

The Taylor micro timescale is defined as $\tau_{T}=\left(2 u^{\prime 2} / a^{2}\right)^{1 / 2}$. The asymptotic behavior of the normalized acceleration variance, $a_{0}=a^{2} /\left(\bar{\epsilon}^{3 / 2} \nu^{-1 / 2}\right)$, at large $R e_{\lambda}$ has not been resolved by current simulations. Although $a_{0}$ is predicted to be constant at large $R e_{\lambda}$ limit by the Kolmogorov 41 theory (see, e.g., Voth et al. 1998; Zaichik et al. 2003), the intermittency corrections to the K41 theory may give it a power-law dependence on $R e_{\lambda}$ (e.g., Borgas 1993). For example, assuming that the temporal statistics of the dissipation rate along Lagrangian trajectories are the same as its spatial statistics in the Eulerian frame (which follows from the ergodic hypothesis and incompressibility; see Borgas (1993)), and using the intermittency theory by She \& Leveque (1994) for the dissipation rate statistics, we find that $a_{0} \propto R e_{\lambda}^{0.133}$ (a similar result was obtained by Borgas (1993) using the log-normal intermittency model). This result is in impressive agreement with one of two formulas that well fit the results from simulations with resolution up to $2048^{3}$ in Yeung et al. (2006),

$$
a_{0}=1.9 \operatorname{Re}_{\lambda}^{0.135}\left(1+85 / \operatorname{Re}_{\lambda}^{1.135}\right)
$$

which goes like $R e_{\lambda}^{0.135}$ at large $R e_{\lambda}$. Therefore, one may expect that $a_{0}=1.9 R e_{\lambda}^{0.135}$ for asymptotically large $R e_{\lambda}$. However, the confirmation of this asymptotic behavior needs future simulations with higher resolutions (Yeung et al. 2006) or more accurate experimental measurements (Voth et al. 1998). We will use eq. (2.18) in our calculations.

Eq. (2.16) approaches $\exp \left(-|\Delta \tau| / T_{L}\right)$ for $z \ll 1$ and $|\Delta \tau| \gg \tau_{T}$. The biexponential form is expected to be better than the single exponential form, $\exp \left(-|\Delta \tau| / T_{L}\right)$, because the Lagrangian correlation is believed to be smooth at small time lag, $|\Delta \tau| \rightarrow 0$. It can be easily shown that $\Phi \sim 1-\Delta \tau^{2} / \tau_{T}^{2}$ for $|\Delta \tau| \ll \tau_{T}$, and thus satisfies the smoothness requirement.

We insert eq. (2.16) into eq. (2.13) to calculate $\mathcal{A}_{i j}$. A lengthy but straightforward integration gives,

$$
\mathcal{A}_{i j}=u^{\prime 2} \delta_{i j} \frac{\left(\Omega_{2}-\Omega_{1}\right)^{2}\left(\Omega_{1} \Omega_{2}+\left(\Omega_{1}+\Omega_{2}\right) \frac{z^{2}}{2}\right)}{\left(\Omega_{1}+\Omega_{2}\right)\left(\Omega_{1}+\Omega_{1}^{2}+\frac{z^{2}}{2}\right)\left(\Omega_{2}+\Omega_{2}^{2}+\frac{z^{2}}{2}\right)}
$$

where $\Omega_{1}$ and $\Omega_{2}$ are defined as $\Omega_{1}=\tau_{p 1} / T_{L}$ and $\Omega_{2}=\tau_{p 2} / T_{L}$. Equation (2.19) correctly reproduces the acceleration term in the S-T limit. When $\Omega_{1}, \Omega_{2} \ll z^{2} / 2$, i.e., $\tau_{p_{1}}, \tau_{p 2} \ll$ $\tau_{T}^{2} /\left(2 T_{L}\right)$ (which is $\sim \tau_{\eta}$ from the K41 phenomenology), we have $\mathcal{A}_{i j} \rightarrow u^{2} \delta_{i j}\left(\Omega_{2}-\right.$ $\left.\Omega_{1}\right)^{2} /\left(z^{2} / 2\right)=\left(\tau_{p 2}-\tau_{p 1}\right)^{2} a^{2} \delta_{i j}$ where the definitions of $\Omega, z$ and $\tau_{T}$ have been used in the last step. As expected, for identical particles, the $\mathcal{A}_{i j}$ term is zero.

Results closely related or directly comparable to eq. (2.19) have been derived from several other models. Williams \& Crane (1983) considered the relative velocities in two limits with $\Omega \ll 1$ and $\Omega \gg 1$, and then gave a "universal" solution by interpolating those two limits. For the small particle limit, they argued that the particle separation (back in time) can be neglected in the calculation of the particle velocity correlation as long as one of the two particles is very small. With this assumption, they find that the 
relative velocity variance, $\left\langle w_{r}^{2}\right\rangle$, is given by (their eq. 19),

$$
\left\langle w_{r}^{2}\right\rangle=u^{\prime 2} \frac{\left(\Omega_{2}-\Omega_{1}\right)^{2}}{\left(\Omega_{1}+\Omega_{2}\right)\left(1+\Omega_{1}\right)\left(1+\Omega_{2}\right)} .
$$

This result corresponds to our result for $\mathcal{A}_{i j}$ because neglecting the backward separation is essentially the same as neglecting the $\mathcal{D}_{i j}$ term. The latter can be justified if one of the two particles, say particle (1), has a very small friction time, i.e., $\tau_{p 1} \rightarrow 0$. In that case, eq. (2.14) is approximately given by $\int_{\infty}^{0} S_{\text {Tij }}\left(\boldsymbol{r} ; 0, \tau^{\prime}\right) \exp \left(\tau^{\prime} / \tau_{p 2}\right) d \tau^{\prime} / \tau_{p 2}$. For $\boldsymbol{r} \rightarrow 0$ as considered by Williams \& Crane (1983), we have $S_{\text {Tij }}\left(\boldsymbol{r}, 0, \tau^{\prime}\right) \rightarrow 0$ and thus $\mathcal{D}_{i j} \rightarrow 0$.

It is obvious that, if $z$ is set to zero, eq. (2.19) for $\mathcal{A}_{i j}$ reduces to eq. (2.20). This is expected because, with $z=0, \Phi$ takes the same single-exponential form used in the derivation of eq. (2.20) by Williams \& Crane (1983). Without the $z$ terms, eq. (2.20) does not reproduce the acceleration term in the S-T limit. Kruis \& Kusters (1997) generalized the model by Williams \& Crane (1983) and used a temporal velocity spectrum that accounts for the acceleration field in the flow (corresponding to a correlation function similar to our eq. (2.16) ) and obtained a formula that gives the acceleration term in eq. (1.1) in the S-T limit.

In $\S 3.2$, we find that for two particles of very different size the contribution to the relative speed from $\mathcal{A}_{i j}$ dominates over that from $\mathcal{D}_{i j}$, and thus eqs. (2.19) or (2.20) can be used to estimate the relative velocities between very different particles (although the justification above for neglecting $\mathcal{D}_{i j}$ is only for the case with at least one tiny particle). However, for similar particles, $\mathcal{D}_{i j}$ is the dominant term, and thus the result for $\Omega \ll 1$ by Williams and Crane is not valid for similar-size particles. In the limit of particles with $\Omega \gg 1$, Williams \& Crane (1983) considered particle separation. We will give more comments on their model in $\S 2.2 .3$ and $\S 4$.

Yuu (1984) derived a formula for the relative velocity with a shear term and an acceleration term. The derivation included an added mass term, $b \partial_{t} \boldsymbol{u}$, in the particle momentum equation (eq. (2.1)). The coefficient $b$, given by $3 \rho_{f} /\left(2 \rho_{p}+\rho_{f}\right)$, is small for solid particles in a gaseous flow and the added mass effect (Kruis and Kuster 1997) is not important. In that case the acceleration term given by Yuu (1984) is exactly the same as eq. (2.20). This means that the acceleration term by Yuu is generally in agreement with our eq. (2.19) for $\tau_{p} \gg \tau_{T}^{2} / T_{L} \sim \tau_{\eta}$. His shear term thus corresponds to our $\mathcal{D}_{i j}$ term, which is the dominant term for similar particles. The problem in Yuu's shear term is that its derivation did not keep track of particle distance in the past, and thus the resulting shear term does not account for particle's memory of the flow velocity difference. This would underestimate the relative velocity between similar particles since the flow velocity difference was larger at earlier times when the particle separation was larger.

With the Lagrangian correlation function, eq. (2.16), the first and third terms in eq. (2.13), representing the velocity variances of particle (1) and $(2),\left(v^{\prime(1)}\right)^{2}$ and $\left(v^{\prime(2)}\right)^{2}$, are given by,

$$
\left(v^{\prime(1)}\right)^{2}=u^{\prime 2} \frac{\Omega_{1}+z^{2} / 2}{\Omega_{1}+\Omega_{1}^{2}+z^{2} / 2}, \text { and }\left(v^{\prime(2)}\right)^{2}=u^{\prime 2} \frac{\Omega_{2}+z^{2} / 2}{\Omega_{2}+\Omega_{2}^{2}+z^{2} / 2} .
$$

For $\Omega_{1}, \Omega_{2} \ll 1$, the particle rms velocity is close to the flow rms velocity $u^{\prime}$. As expected, in the limit $\Omega_{1}, \Omega_{2} \gg 1$, the sum of the two terms reproduces eqs. (1.2) and (1.3) for the relative velocity of large particles. This means that, in order to build a model that gives correct prediction in the limit of very large $\tau_{p}$, one needs to guarantee that the sum of the other two terms in the model, i.e., the 2 nd term in eq. (2.13) and $\mathcal{D}_{i j}$ (both from the cross correlation of particle velocities), approaches zero as $\tau_{p} \rightarrow \infty$. More specifically, 
the sum of those two terms has to approach zero faster than $1 / \tau_{p}$ so that they do not dominate over $\left(v^{\prime(1)}\right)^{2}$ and $\left(v^{\prime(2)}\right)^{2}$ given by eq. (2.21). We will show that the model presented in the next subsection for the $\mathcal{D}_{i j}$ term does satisfy this constraint, and hence eqs. (1.2) and (1.3) are correctly reproduced in our model.

Finally, we point out that the assumption we adopted that the temporal correlation of the flow velocity on an inertial particle's trajectory can be approximated by the Lagrangian correlation function may be invalid for large $\tau_{p}$. The trajectory of a large particle can be very different from that of a tracer particle. For example, if $\tau_{p} \gtrsim T_{L}$ or $T_{E}$, the particle may not move significantly as the flow "sweeps" by. Thus the correlation of the flow velocity along the trajectory of such a heavy particle may be better approximated by the Eulerian temporal correlation. This means that the flow velocity correlation on a particle's trajectory could make a transition from Lagrangian-like to Eulerian-like as $\tau_{p}$ increases. In that case, replacing the Lagrangian correlation timescale $T_{L}$ in eq. (2.21) by the Eulerian correlation timescale, $T_{E u}$, would give a better estimate for the rms velocity of large particles. $T_{L}$ in eq. (1.2) from Abrahamson (1975) should also be replaced by $T_{E u}$. If the Eulerian correlation timescale is larger than $T_{L}$ (e.g., Yeung \& Pope 1989; Kaneda \& Gotoh 1991), our model would underestimate the relative speed by a factor of $\left(T_{E u} / T_{L}\right)^{1 / 2}$ in the limit of $\tau_{p} \gg T_{L}$. A numerical study of the flow velocity correlation on particles' trajectory as a function of $\tau_{p}$ would be useful to improve our model.

\subsection{Modeling $\mathcal{D}_{i j}$}

In order to evaluate the generalized shear term, $\mathcal{D}_{i j}$, we need to model the trajectory structure tensor, $S_{\mathrm{T} i j}$, which has not been directly measured. The tensor can be formally written as

$$
S_{\mathrm{T} i j}=\iint\left\langle\delta u_{i}^{(p)}(\tau) \delta u_{j}^{(p)}\left(\tau^{\prime}\right) \mid \boldsymbol{\rho}, \boldsymbol{\rho}^{\prime}\right\rangle P\left(\boldsymbol{\rho}, \boldsymbol{\rho}^{\prime} ; \boldsymbol{r}, \tau, \tau^{\prime}\right) d \boldsymbol{\rho} d \boldsymbol{\rho}^{\prime}
$$

where $P$ is the joint probability distribution of the particle separations, $\boldsymbol{\rho}$ and $\boldsymbol{\rho}^{\prime}$, at $\tau$ and $\tau^{\prime}$, respectively, and we have used $\delta u_{i}^{(p)}(\tau)\left(\equiv u_{i}\left(\mathbf{X}^{(\mathbf{1})}(\tau)\right)-\mathbf{u}_{\mathbf{i}}\left(\mathbf{X}^{(\mathbf{2})}(\tau)\right)\right)$ and $\delta u_{j}^{(p)}\left(\tau^{\prime}\right)$ to denote the flow velocity difference "seen" by the two particles. The ensemble average term in the integrand is the velocity difference correlation conditioned on the particle separations. A series of assumptions need to be made for the estimate of $S_{\mathrm{T} i j}$ since both the conditional correlation and the joint probability of the particle separations are unknown.

The conditional correlation depends on both the separations, $\boldsymbol{\rho}$ and $\boldsymbol{\rho}^{\prime}$, and the two times, $\tau$ and $\tau^{\prime}$. It may also have a direct dependence on $\boldsymbol{r}$, in addition to that through $\boldsymbol{\rho}$ and $\boldsymbol{\rho}^{\prime}$. This possible dependence is neglected here. We assume that the amplitude of the velocity difference $\delta u_{i}^{(p)}(\tau)$ on particles' trajectories can be approximated by the Eulerian velocity difference, $\delta u_{i}(\boldsymbol{\rho}, \tau)$, across the separation $\boldsymbol{\rho}$. This assumption is similar to the independence hypothesis by Corrsin (1959) (note the similarity between eq. (5) in Corrsin (1959) and our eq. (2.22); see also Shlien \& Corrsin (1974)). Assuming that the displacement of a fluid particle (from its initial position) is statistically independent from the particle's current velocity, Corrsin (1959) gave a relation between the Lagrangian correlation function and the Eulerian correlation. The hypothesis by Corrsin essentially neglects a constraint between the particle displacement and the velocity along the Lagrangian trajectory (i.e., the latter equals the time derivative of the former). In the case of inertial particles, the constraint neglected in our approximation is $d^{2} \boldsymbol{\rho} / d t^{2}=\left(\delta \boldsymbol{u}^{(p)}-d \boldsymbol{\rho} / d t\right) / \tau_{p}$. The existence of this constraint could give rise to a statistical correlation between $\delta \boldsymbol{u}^{(p)}$ and $\rho$. For example, a larger particle separation at a given time suggests a larger (on average) velocity difference in the past, and perhaps 
a higher possibility of a large velocity difference right at that time. Therefore, relative to the Eulerian velocity difference across a fixed separation, the velocity difference along the particles' trajectories may have a stronger dependence on the particle separation. Setting $\delta u_{i}^{(p)}$ to the Eulerian velocity difference, $\delta u_{i}(\boldsymbol{\rho})$, could thus underestimate the dependence of $\delta u_{i}^{(p)}$ on $\rho$. The uncertainty and reliability of our assumption here are subject to tests by future numerical experiments.

From the assumption above, we have $\left\langle\delta u_{i}^{(p)}(\tau) \delta u_{j}^{(p)}\left(\tau^{\prime}\right) \mid \boldsymbol{\rho}, \boldsymbol{\rho}^{\prime}\right\rangle \simeq\left\langle\delta u_{i}(\boldsymbol{\rho}, \tau) \delta u_{j}\left(\boldsymbol{\rho}^{\prime}, \tau^{\prime}\right)\right\rangle$. The correlation of the Eulerian velocity differences at two times is also unknown and further approximations are needed. We first assume that it can be written as a product of a separation dependence term and a time-lag dependence term. The separation dependence term is then assumed to take the form of the Eulerian structure function of the flow, $S_{i j}$. Note that, the conditional correlation actually depends on two separations, $\boldsymbol{\rho}$ and $\boldsymbol{\rho}^{\prime}$, but for simplicity we will approximate it by $S_{i j}(\boldsymbol{R})$, at a single separation, $\boldsymbol{R}$, characteristic of the particle distances between $\tau$ and $\tau^{\prime}$. The choice for the separation $\boldsymbol{R}$ as a function of $\boldsymbol{\rho}$ and $\boldsymbol{\rho}^{\prime}$ will be discussed later. As a function of the two stochastic vectors, $\boldsymbol{R}$ is also stochastic.

The time-lag dependence accounts for the temporal correlation between the flow velocity differences "seen" by the two particles. This correlation depends on the persistence of the "structure" in question, which is a function of the "structure size". Here the size is essentially the distance between the two particles. Associated with each structure, there is a correlation timescale, $T_{R}$. To estimate $T_{R}$, one needs to pick up a size to characterize the structure that corresponds to particle distances at time between $\tau$ and $\tau^{\prime}$. We will take the size to be the same as the particle separation $R$ to characterize the separation dependence. We point out that there is no physical motivation for this particular choice, and that it could be better to choose the distance, $R_{m}$, at the earlier one of the two times $\tau$ and $\tau^{\prime}, \min \left(\tau, \tau^{\prime}\right)$, assuming the persistency of a structure is determined by its initial size. The difference in the results from the two choices will be discussed in $\S 3$. The time lag dependence is assumed to take the same function form as eq. (2.16) for the Lagrangian temporal correlation in $\S 2.2$. Namely, we set the time-lag dependence to be $\Phi\left(\tau-\tau^{\prime} ; \tau_{T R}(R), T_{R}(R)\right)$, where the Lagrangian timescale, $T_{L}$, in eq. (2.16) has been replaced by $T_{R}(R)$ and $\tau_{T}$ by $\tau_{T R}(R)$. The timescale $\tau_{T R}(R)$ is an analogue of the Taylor micro timescale for a structure of size $R$ (see Zaichik et al. 2003, 2006).

The conditional correlation is now approximated by $S_{i j}(\boldsymbol{R}) \Phi\left(\tau-\tau^{\prime} ; \tau_{T R}(R), T_{R}(R)\right)$. In order to estimate the trajectory structure function, in principle one needs to integrate the conditional correlation over the distribution $P\left(\boldsymbol{\rho}, \boldsymbol{\rho}^{\prime}\right)$ (eq. 2.22), or equivalently over the distribution, $P(\boldsymbol{R})$, of $\boldsymbol{R}$ (in our approximation the conditional correlation depends on the separations $\boldsymbol{\rho}$ and $\boldsymbol{\rho}$ only through $\boldsymbol{R}$ ). We take a simple approach here. We set the integral to be equal to the conditional correlation at a distance corresponding to the rms of $\boldsymbol{R}$. This means that the particle distance at a given time is assumed to be single-valued, i.e., the distribution $P(R)$ is a delta function at the rms of $(R)$. A rough justification of this approximation will be given in $§ 2.3 .3$. The direction of $\boldsymbol{R}$ probably has a random distribution due to the turbulent dispersion, and we will average the structure tensor $S_{i j}(\boldsymbol{R})$, over the direction distribution of $\boldsymbol{R}$.

For simplicity in notations, hereafter we will use $R$ to denote the rms length of $\boldsymbol{R}$, i.e., $R^{2}=\left\langle\boldsymbol{R}^{2}\right\rangle$ (note that this is different from the conventional notation that $R$ denotes the length of a vector $\boldsymbol{R}$, i.e., $R^{2}=\boldsymbol{R}^{2}$, without ensemble averaging). Similarly, $\rho(\tau)$ will denote the rms length of $\boldsymbol{\rho}(\tau)$. We refer to $R$ and $\rho$ as "rms distance" or simply "distance", while using "separation" for the corresponding stochastic vectors. 
The approximations for the trajectory structure tensor are now complete,

$$
S_{\text {Tij }}\left(\boldsymbol{r} ; \tau, \tau^{\prime}\right) \simeq\left\langle S_{i j}(\boldsymbol{R})\right\rangle_{\mathrm{ang}} \Phi\left(\tau-\tau^{\prime} ; \tau_{T R}(R), T_{R}(R)\right)
$$

where the ensemble average for $S_{i j}(\boldsymbol{R})$ is over the direction distribution of $\boldsymbol{R}$. The angular average will be carried out in $\S 2.3 .3$.

Finally we need to specify the rms distance $R$ as a function of $\rho(\tau)$ and $\rho\left(\tau^{\prime}\right)$. If the flow velocity difference scales with the distance as a power law, which is probably the case in well-developed, homogeneous and isotropic turbulence, a good choice would be

$$
R\left(\tau, \tau^{\prime}\right)=\left(\rho(\tau) \rho\left(\tau^{\prime}\right)\right)^{1 / 2} .
$$

We note that, in their assumption for the Lagrangian structure tensor, Zaichik \& Alipchenkov (2003) apparently set $R$ to be the particle separation at the earlier time of $\tau$ and $\tau^{\prime}$. We argue that eq. (2.24) is probably a better assumption because $S_{\mathrm{T} i j}$ is expected to be zero if either $\rho(\tau)$ or $\rho\left(\tau^{\prime}\right)$ is zero.

The assumption, eq. (2.24), is expected to be valid when $\rho$ and $\rho^{\prime}$ are in the same length scale subrange since in that case the scaling of velocity difference across the two separations follows the same power-law (see, §2.3.1). On the other hand, if $\rho$ and $\rho^{\prime}$ are in different subranges, the structure function across $R$ defined by eq. (2.24) may not correctly represent the product of the velocity difference amplitudes across $\rho$ and $\rho^{\prime}$. Fortunately, we find that this does not significantly affect our prediction of the relative speed based on a consideration of the $\Phi$ term in the trajectory structure tensor (the temporal correlation of the velocity differences). The $\Phi$ term gives an exponential cutoff when the time lag is large, i.e., for very different $\tau$ and $\tau^{\prime}$. Note that, if $\rho$ and $\rho^{\prime}$ are in different subranges, in general $\tau$ and $\tau^{\prime}$ would also be very different (although it is possible that the particle pair experiences a large separation change during a short time interval, these extreme events should be rare, and not affect the low order statistics, i.e., the second order particle structure function, we study here). Therefore, the exponential cutoff from the $\Phi$ term would suppress the contribution from very different $\rho$ and $\rho^{\prime}$ to the integral for $\mathcal{D}_{i j}$. In other words, the temporal decorrelation of the velocity difference over large time lags suggests that the main contribution to $\mathcal{D}_{i j}$ is probably from similar $\rho$ and $\rho^{\prime}$, where eq. (2.24) is valid.

\subsubsection{The Flow Structure Tensor and the Timescales}

In homogeneous and isotropic turbulence, the Eulerian structure tensor at a separation $\boldsymbol{l}$ can be written as (e.g., Monin \& Yaglom 1975),

$$
S_{i j}(\boldsymbol{l})=S_{n n}(l) \delta_{i j}+\left(S_{l l}(l)-S_{n n}(l)\right) \frac{l_{i} l_{j}}{l^{2}}
$$

where $S_{n n}$ and $S_{l l}$ are, respectively, transverse and longitudinal structure functions. For an incompressible velocity field, they are related by,

$$
S_{n n}(l)=S_{l l}(l)+\frac{l}{2} \frac{d S_{l l}(l)}{d l} .
$$

The longitudinal structure function, $S_{l l}$, in different ranges of length scales is given as follows.

As mentioned in $\S 1$, in the viscous subrange, $S_{l l}$ is given by,

$$
S_{l l}(l)=\frac{\bar{\epsilon}}{15 \nu} l^{2}, \text { for } l \lesssim \eta .
$$

In the inertial subrange, we have,

$$
S_{l l}(l)=C(\bar{\epsilon} l)^{2 / 3}, \text { for } \eta \lesssim l \lesssim L_{1}
$$


where the coefficient $C$ for the velocity scaling in the inertial range is believed to be universal and will be set to $C=2$ (Monin \& Yaglom 1975; Zaichik \& Alipchenkov 2003).

For $l$ larger than the integral scale, $S_{l l}$ is constant,

$$
S_{l l}(l)=2 u^{\prime 2}, \text { for } l \gtrsim L_{1} .
$$

The characteristic scale at which $S_{l l}$ switches from the viscous-range scaling to the inertial-range scaling can be obtained by equating eqs. (2.27) and (2.28). This gives a transition scale of $(15 C)^{3 / 4} \eta$, which is about $13 \eta$ for $C=2$. It is consistent with the simulation results given in Ishihara et al. (2009), where the switch occurs at about a few tens of Kolmogorov scale, between $\eta$ and the Taylor micro scale $\lambda$. The scaling changes from eq. (2.28) to eq. (2.29) at $l \simeq(2 / C)^{3 / 2} L$ where $L=u^{\prime 3} / \bar{\epsilon}$, as defined earlier. This is also in general agreement with Fig. 7b in Ishihara et al. (2009), which shows that $S_{l l}$ becomes constant at $\simeq 0.5-3 L_{1}\left(L_{1} \simeq 0.4 L\right)$.

We will use the following formula from Zaichik et al. (2006) to connect the velocity scalings in different subranges,

$$
S_{l l}(l)=2 u^{\prime 2}\left[1-\exp \left(-\frac{l}{(15 C)^{3 / 4} \eta}\right)\right]^{4 / 3}\left(\frac{l^{4}}{l^{4}+(2 / C)^{6} L^{4}}\right)^{1 / 6} .
$$

The transverse structure function $S_{n n}$ then follows from eq. (2.26).

The timescale $T_{R}$ as a function of the separation in eq. (2.23) has different scalings in the three subranges above as well. The theoretical model by Lundgren (1981) found that in the viscous range,

$$
T_{R}(l)=\sqrt{5} \tau_{\eta}, \text { for } l \lesssim \eta
$$

which was later confirmed by numerical simulations (Girimaji \& Pope 1990).

In the inertial range, the similarity argument suggests that,

$$
T_{R}(l)=C_{2} \bar{\epsilon}^{-1 / 3} l^{2 / 3}, \text { for } \eta \lesssim l \lesssim L_{1} .
$$

Following Zaichik et al. (2006), we will take the coefficient $C_{2}=0.3$ in our calculations. Eqs. (2.31) and (2.32) connect at $\simeq\left(\sqrt{5} / C_{2}\right)^{3 / 2} \eta$, which is $\simeq 20 \eta$ for $C_{2}=0.3$. This is a little larger than the corresponding transition scale $(13 \eta)$ for $S_{l l}$.

For $l \gg L_{1}$, the flow velocities across $l$ are independent, $T_{R}$ is thus expected to be the correlation timescale of the velocity along the trajectory of each particle, which is approximately given by the Lagrangian correlation timescale, $T_{L}$ (see Zaichik \& Alipchenkov 2003),

$$
T_{R}(l)=T_{L}, \text { for } l \gtrsim L_{1} .
$$

This connects to the inertial-range scaling at about $\bar{\epsilon}^{1 / 2}\left(T_{L} / C_{2}\right)^{3 / 2}$, which is $\simeq L$ using $C_{2}=0.3$ and $T_{L} \simeq 0.3 u^{\prime 2} / \bar{\epsilon}$.

Similar to the case for $S_{l l}$, a formula is used to connect $T_{R}$ in different subranges,

$$
T_{R}(l)=T_{L}\left[1-\exp \left(-\left(\frac{C_{2}}{\sqrt{5}}\right)^{3 / 2} \frac{l}{\eta}\right)\right]^{-2 / 3}\left(\frac{l^{4}}{l^{4}+T_{L}^{6}(\bar{\epsilon})^{2} / C_{2}^{6}}\right)^{1 / 6}
$$

which is again adopted from Zaichik et al. (2006).

By analogy to the definition of the Taylor micro timescale, $\tau_{T}$, we estimate $\tau_{T R}(l)$ by $\tau_{T R}^{2} \simeq 2\left\langle\delta u(l)^{2}\right\rangle /\left\langle\delta a(l)^{2}\right\rangle$ where $\delta u(l)$ and $\delta a(l)$ are the velocity and acceleration difference across a distance $l$. This formula is simply a generalization of $\tau_{T}$ for the oneparticle Lagrangian correlation to that for two-particle Lagrangian structure function. The correlation length scale of the acceleration field is expected to be short, probably $\sim \eta$. In that case, for $l$ in the inertial range $\left\langle\delta a(l)^{2}\right\rangle \simeq 2 a^{2}$ with $a$ the rms acceleration 
and thus $\tau_{T R} \propto \delta u(l) \sim l^{1 / 3}$. From $T_{R}(l) \sim l^{2 / 3}$, we have $\tau_{T R}(l) \propto T_{R}(l)^{1 / 2}$. In our calculations, we will use $\tau_{T R}=\tau_{T}\left(T_{R} / T_{L}\right)^{1 / 2}$. Note that this formula gives $\tau_{T R}=\tau_{T}$ for $l \gtrsim L$, as expected. It gives a constant $\tau_{T R}$ for $l \rightarrow 0$. Zaichik et al. (2003, 2006) assumed that $\tau_{T R}(l)=\left(\tau_{T} / T_{L}\right) T_{R}(l)=z T_{R}(l)$ without providing a physical motivation. Our calculations find that the two different assumptions for $\tau_{T R}$ do not give significant difference in the predicted relative speed.

\subsubsection{Particle Pair Dispersion}

We now consider the rms distance, $\rho(\tau)$, of two particles as a function of time $\tau$, which is needed to evaluate $R$ (eq. (2.24)). As mentioned earlier, the specific question we ask here is how particles separate from each other backward in time, given their separation, $\boldsymbol{r}$, at time zero (we will also refer to $r$ as the initial distance for the backward dispersion from the viewpoint of the reversed time direction, although with normal time direction it is the final distance of the two particles in question).

The study of turbulent dispersion of inertial particles started only recently (Bec et al. 2007; Fouxon \& Horvai 2008; Bec et al. 2009a), Bec et al. (2009a) gave a detailed report of simulation results for the separation behavior forward in time. They found that there are two temporal regimes with different separation behaviors: a transient regime representing the relaxation of the particle velocity toward the flow velocity, and a later regime where the particle pairs separate in a similar way as tracer particles. The transient regime lasts for about a friction timescale. For $S t \gtrsim 3$, a ballistic separation is found in the transient regime and the separation speed is equal to the initial velocity difference. The ballistic separation is due to the particles' memory for a period of $\sim \tau_{p}$. In the later phase, the particle separation is found to follow the Richardson-Obukhov separation law.

Although the study by Bec et al. (2009a) is for dispersion forward in time, their results provide a very useful guideline for us, because we are are not aware of any investigations for the backward dispersion of inertial particles. A separation behavior similar to that found by Bec et al. (2009a) will be used in our calculations (§3.1.3). We will show that a combination of an earlier ballistic phase and a later tracer-like phase gives quite good fit to the numerical simulation results for the relative speed by Wang et al. (2000). For St $\lesssim 1$, the particle separation appears to increase slower than linearly with time in the transient phase according to Fig. 5 in Bec et al. (2009a). However, no function fit to the separation behavior in this regime is given by Bec et al. (2009a). For simplicity, we will assume that the separation is ballistic for the early phase of all particles. This assumption gives rise to uncertainty in our prediction for the relative speed for particles with $S t \lesssim 1$.

In order to understand the results of our model with the two-phase separation, we need to know the effect of each phase, and thus we first consider two simplified cases assuming a complete ballistic behavior (§3.1.1) and a complete tracer-like behavior (§3.1.2), respectively. The simplified cases give a very useful illustration for the effect of particle separation on the predicted relative speed. For example, for both ballistic and tracerlike separation behaviors, the relative speed as a function of the Stokes number can be explained from an approximate analysis of the integral equation for $\mathcal{D}_{i j}$. In particular, the analysis gives physical insights on the scaling of the relative speed with the Stokes number in the inertial range for the monodisperse case. Once the two simplified cases are understood, it is straightforward to interpret the prediction of our model with the more realistic separation behavior by a combination of two phases (§3.1.3).

For ballistic motions, the particle distance goes linearly with time. Given the particle distance, $r$, at time zero, the separation as a function of $\tau$ is,

$$
\rho^{2}(\tau)=r^{2}+\left\langle w^{2}\right\rangle \tau^{2}
$$


where $\left\langle w^{2}\right\rangle=\left\langle\left(\boldsymbol{v}^{(1)}-\boldsymbol{v}^{(2)}\right)^{2}\right\rangle=S_{\mathrm{p} i i}(\boldsymbol{r})$ is the $3 \mathrm{D}$ relative velocity variance of the two particles at time zero. The separation speed at any time is taken to be the same as that at time zero. Recall that in our notation $\rho(\tau)$ is the rms of $\boldsymbol{\rho}(\tau)$. The relative velocity variance, $\left\langle w^{2}\right\rangle$, in eq. (2.35) is unknown and is directly related to the radial relative speed under pursuit. We will build an implicit equation for $\left\langle w^{2}\right\rangle$ and $\left\langle w_{r}^{2}\right\rangle$ in $\S 3$, which is then solved self-consistently.

The exponential cutoffs in the integrand of eq. (2.14) imply that the primary contribution to $\mathcal{D}_{i j}$ is from $-\tau_{p 1} \lesssim \tau \leqslant 0$ and $-\tau_{p 2} \lesssim \tau^{\prime} \leqslant 0$. This means that if the ballistic behavior lasts for about $\tau_{p}$ (Bec et al. 2009a), then using eq. (2.35) at all times when integrating eq. (2.14) may give an acceptable order-of-magnitude estimate for $\mathcal{D}_{i j}$, even though the separation is not ballistic at later time.

Williams \& Crane 1983) (and also Kruis \& Kusters 1997) take the particle motions to be ballistic in their calculations for the limit with large particles $\left(\tau_{p} \gg T_{L}\right)$. Assuming the velocity correlation between two particles can be neglected for the purpose of estimating the particle separation, they set the linear separation rate to $\left(\left(v^{\prime(1)}\right)^{2}+\left(v^{\prime(2)}\right)^{2}\right)^{1 / 2}$. We note that this assumed separation rate may be a good approximation only for very large particles. In the case of small to intermediate particles, the velocities of nearby particles are correlated and the separation rate is smaller than given by Williams \& Crane (1983). Using their separation speed for those particles would overestimate the relative speed because both $S_{i j}$ and $\Phi$ in eq. (2.23) increase with the particle distance, as can be seen from eqs. (2.30) and (2.34).

In the second simplified case, we will consider the separation behavior similar to that of tracer particle pairs (see Falkovich et al. 2001; Salazar \& Collins 2009, for detailed reviews on the pair dispersion of tracers). We are particularly interested in the effect of Richardson-Obukhov separation (the R-O separation hereafter) phase found in the forward dispersion of inertial particle pairs. The R-O separation law is written as

$$
\rho^{2}(\tau) \propto g \bar{\epsilon}|\tau|^{3}
$$

where the dimensionless coefficient $g$ is known as the Richardson constant. Bec et al. (2009a) did not give best-fit values for $g$ in the late-phase separation. Apparently $g$ needs to be adjusted to fit the simulation results (Bec et al. (2009a)), and it probably has a Stokes number dependence. Since the value of $g$ for inertial particles is unknown, we will first use $g$ measured for tracer pairs as a reference. For tracers, theoretical models and direct numerical simulations by Sawford et al. (2005) show that the dispersion backward in time is significantly faster than the forward dispersion. Experimental measurements by Berg et al. (2006) found that $g=0.55$ for the forward dispersion, and $g=1.15$ for the dispersion backward in time. We will take $g=1.15$ as a reference value since it is the backward dispersion that is relevant in our problem. In $\S 3.1 .3$, we show that a two-phase separation with $g \sim 1$ in our model gives good fit to the numerical results for the relative speed.

When the particle separation becomes larger than the integral scale, the flow velocities "seen" by the particle pair are uncorrelated and the separation is expected to be diffusive. Thus we will switch from the R-O law to the diffusive separation when the particle distance exceeds $L$, i.e., we set $\rho^{2}(\tau) \simeq 2 D|\tau|$ for $\rho \geqslant L$ where the coefficient $D$ is given by $D=6 u^{\prime 2} T_{L}$. Note that $D$ here is for the $3 \mathrm{D}$ diffusion, and it is twice larger than $D$ for 1-particle diffusion (from the Taylor theorem) because the rms relative velocity of two faraway particles is $2 u^{\prime 2}$. We find that the exact separation behavior in the range $\rho \gtrsim L$ in the diffusive regime is not important for the integration of eq. (2.14) because $S_{i j}$ and $T_{R}$ in eq. (2.23) becomes constant in this range of the particle distance. 
The smallest initial separation considered by Bec et al. (2009a) is about $1 \eta$. For an initial separation much below $\eta$, there probably exists an initial exponential separation phase (similar to that of tracer pairs at small separations). The Lyapunov exponents for particles with $S t \lesssim 2$ have been computed from simulation by Bec et al. (2006). Although the exponential regime would also exist for larger particles as implied by the chaoticity of the dynamics (see, e.g., Bec et al. (2007) for a theoretical model which predicts that the Lyapunov exponent decreases as $S t^{-2 / 3}$ for large $S t$ ), its relevance at finite (but below $\eta$ ) scale separation is questionable at least in the large $S t$ limit. Here, we will not consider the exponential phase, since it is unknown how long it lasts and how it connects with the later phases. In most of our calculations we will give results for $r \sim \eta$. In the simulations by Wang et al. (2000) and Zhou et al. (2001) that we will use to test our model, the relative speed is measured at a distance of $\eta$, thus it is sufficient to use Bec et al.'s result as a guideline in the comparison with those simulation results. It is straightforward to incorporate an exponential phase into our model, and once the detailed separation behavior of all particles at distances well below $\eta$ is known, our model can predict the relative speed at any separation.

Based on our physical picture, a careful consideration of the particle pair dispersion is necessary for an accurate estimate of the relative velocity. It is likely that the physics of turbulent dispersion of particle pairs is implicitly incorporated into the quite successful model by Zaichik and collaborators. In fact, their equation for the joint pdf of the particle separation and the relative velocity can be regarded as one for the pdf of the particle distance when integrated over the relative velocity (phase) space. For a comparison of our results with Zaichik et al.'s model, it is useful to see how tracer pairs separate in their framework, i.e., the prediction for particle separation from their formulation in the limit $\tau_{p} \rightarrow 0$. In this limit, the joint pdf equation can be reduced to an equation for the pdf of the particle distance. The pdf equation turns out to be in the same form as that suggested by Richardson (1926). With structure functions and timescales given in $\S 2.3$ (same as in Zaichik et al. (2006)), the equation suggests that the Richardson constant, $g$, is about 3 , significantly larger than measured from experiments and numerical simulations. It is not clear whether and how the quasi-normal assumption made by Zaichik et al. to close the moment equations of the joint pdf equation may affect the particle dispersion, or what it physically corresponds to regarding the separation.

\subsubsection{Average over the Direction of $\boldsymbol{R}$}

We calculate the average of $S_{i j}$ over the direction of $\boldsymbol{R}$ in eq. (2.23). For $\boldsymbol{R}$ at any given time, we define a separation difference $\Delta \boldsymbol{R}=\boldsymbol{R}-\boldsymbol{r}$, the change of the separation from $\boldsymbol{r}$ at time zero. The direction of the separation difference is expected to be completely random if the flow velocity is statistically isotropic. This means that $\left\langle\Delta R_{i} \Delta R_{j}\right\rangle_{\text {ang }}=\frac{\Delta R^{2}}{3} \delta_{i j}$. We set $\boldsymbol{l}$ in eq. (2.25) to be $\boldsymbol{R}=\boldsymbol{r}+\Delta \boldsymbol{R}$ and take the average over the direction of $\Delta \boldsymbol{R}$. A rigorous derivation of this average needs to consider the dependence of $S_{i j}(\boldsymbol{R})$ on $\Delta \boldsymbol{R}$ through the length $R=|\boldsymbol{r}+\Delta \boldsymbol{R}|$ (e.g., in $S_{l l}$ and $S_{n n}$ ) and that through the tensor $R_{i} R_{j}$ simultaneously. However, the derivation is very complicated and cannot be done analytically. For simplicity, we neglect the dependence through $R$ in the averaging process and only consider the average of $R_{i} R_{j}$ over the $\Delta \boldsymbol{R}$ direction. With this approximation, we find,

$$
\left\langle S_{i j}(\boldsymbol{R})\right\rangle_{\mathrm{ang}}=\left[\left(\frac{2}{3}+\frac{r^{2}}{3 R^{2}}\right) S_{n n}(R)+\left(\frac{1}{3}-\frac{r^{2}}{3 R^{2}}\right) S_{l l}(R)\right] \delta_{i j}+\left(S_{l l}(R)-S_{n n}(R)\right) \frac{r_{i} r_{j}}{R^{2}}
$$

where we have used $\left\langle\Delta R_{i} r_{j}\right\rangle_{\text {ang }}=0$ and $(\Delta R)^{2}=R^{2}-r^{2}$. 
The generalized shear term, $\mathcal{D}_{i j}$, then follows from eqs. (2.14), (2.23) and (2.37),

$$
\begin{aligned}
\mathcal{D}_{i j}(r)=\int_{-\infty}^{0} \frac{d \tau}{\tau_{p 1}} \int_{-\infty}^{0} & \frac{d \tau^{\prime}}{\tau_{p 2}}\left\{\left[\left(\frac{2}{3}+\frac{r^{2}}{3 R^{2}}\right) S_{n n}(R)+\left(\frac{1}{3}-\frac{r^{2}}{3 R^{2}}\right) S_{l l}(R)\right] \delta_{i j}\right. \\
& \left.+\left(S_{l l}(R)-S_{n n}(R)\right) \frac{r_{i} r_{j}}{R^{2}}\right\} \times \Phi\left(\tau-\tau^{\prime} ; \tau_{T R}(R), T_{R}(R)\right) \\
& \times \exp \left(\frac{\tau}{\tau_{p 1}}\right) \exp \left(\frac{\tau^{\prime}}{\tau_{p 2}}\right) .
\end{aligned}
$$

We will numerically integrate this equation in $\S 3$ using the structure functions $S_{l l}$ and $S_{n n}$ and the timescales $\tau_{T R}$ and $T_{R}$ given in $\S 2.3 .1$, and particle pair separation laws for $\rho$ and $R$ given in $\S 2.3 .2$.

In eq. (2.38), the structure functions, the timescale $T_{R}$, and hence $\Phi$ (eq. (2.16)) increase with $R$, which increases with $|\tau|$ and $\left|\tau^{\prime}\right|$. Together with the exponential cutoffs, this suggests that the integrand in eq. (2.38) peaks at $\tau \simeq-\tau_{p 1}$ and $\tau^{\prime} \simeq-\tau_{p 2}$. Therefore, the main contribution to the integral is from $\tau \sim-\tau_{p 1}$ and $\tau^{\prime} \sim-\tau_{p 2}$ (if $\tau_{p 1}$ and $\tau_{p 2}$ are not very different) and an important factor to determine the value of the integral is the distance, $R$, at $\tau \sim-\tau_{p 1}$ and $\tau^{\prime} \sim-\tau_{p 2}$, which we will refer to as the primary distance. If $\tau_{p 1}$ and $\tau_{p 2}$ are both very small so that the primary distance is close to $r, \mathcal{D}_{i j}$ would approach $S_{i j}(r)$ as expected for the S-T limit. If the primary distance is much larger than the particle distance at time zero, $r$, the $r^{2} / R^{2}$ terms in eq. (2.38) can be neglected and $\mathcal{D}_{i j} \propto \delta_{i j}$ (meaning that $\mathcal{D}_{l l}=\mathcal{D}_{n n}=\mathcal{D}_{i i} / 3$ ).

If $\tau_{p 1}$ and $\tau_{p 2}$ are in the large limit and the primary distance is much larger than the length scale $L$, then typically the structure tensor in the integral in eq. (2.38) is $\sim 2 u^{\prime 2} \delta_{i j}$ (see eq. (2.29)), and the $\Phi$ term would be the same as eq. (2.16) because $T_{R}=T_{L}$ (eq. (2.33)) for $R \gtrsim L$. This means that the integrand of eq. (2.38) would be the same as that in the 2 nd term on the r.h.s. of eq. (2.13) (with $B_{\text {Tij }}$ given by the assumptions in $\S 2.2$ ) for the range of $\tau$ and $\tau^{\prime}$ with $R\left(\tau, \tau^{\prime}\right) \gtrsim L$. On the other hand, at smaller values of $|\tau|$ and $\left|\tau^{\prime}\right|$ (with $R<L$ ), the integrand for $\mathcal{D}_{i j}$ is smaller than that in the latter. Thus $\mathcal{D}_{i j}$ given by (2.38) does not exactly cancel out the 2 nd term on the r.h.s. of eq. (2.13). We find both numerically and analytically that the sum of the two terms decreases faster than $1 / \tau_{p}$ in the limit $\tau_{p} \rightarrow \infty$ (it goes like $\tau_{p}^{-3 / 2}$ for the assumption of the ballistic separation and like $\sim \tau_{p}^{-2}$ for the tracer-like separation behavior). Therefore, in the limit of large friction timescales our model satisfies the constraint discussed at the end of $\S 2.1$, namely, a good model needs to give a particle velocity correlation that decreases with $\tau_{p}$ faster than the other terms.

As discussed earlier, we used the rms distance, $\rho$ and $R$, in our estimate of the trajectory structure tensor, $S_{\mathrm{T} i j}$, while a rigorous derivation needs to consider the probability distribution function of the separation and take the average of $S_{\mathrm{T} i j}$ over this distribution. Here we give a justification for this approximationt $t$. First, if the separation is in the viscous range, $R \lesssim \eta, S_{i j}$ has a quadratic dependence on the separation (eq. (2.27)) and $\Phi$ is independent of $R$ (eq. (2.31)). This suggests that the approximation is exact since the average of the quadratic dependence over the separation distribution is exactly the square of the rms distance. If $R$ is in the inertial range, then $S_{i j} \propto R^{2 / 3}$, and, roughly speaking, the $\Phi$ term provides another factor of $R^{2 / 3}$ (from the timescale $T_{R}(R)$, eq. (2.32), over which the flow velocity difference "seen" by the particles is correlated).

$\dagger$ In the justification argument, $R$ is taken to be a stochastic variable, the length of the stochastic vector $\boldsymbol{R}$, although at all other places in the paper $R$ refers to the rms length of $\boldsymbol{R}$. The rms distance will be written explicitly as $\left\langle R^{2}\right\rangle^{1 / 2}$ in the argument here. This change of notation is only for the present paragraph. 
Therefore, using a single rms distance to replace the distance pdf would overestimate $S_{\text {Tij }}$ (and hence $\mathcal{D}_{i j}$ ) by a factor of $\simeq\left\langle R^{2}\right\rangle^{2 / 3} /\left\langle R^{4 / 3}\right\rangle$. If the $\boldsymbol{R}$ pdf is $3 \mathrm{D}$ Gaussian as in the relative diffusion model by Batchelor (1952), this factor is only 1.07. Numerical simulations (e.g., Boffetta \& Sokolov 2002) show that the separation pdf of tracer pairs is highly non-Gaussian with a very broad tail. The pdf is found to be well fit by the solution of the pdf equation proposed by Richardson (1926). With this broader pdf, we find the factor is larger, $\simeq 1.20$. Because $S_{i j}$ and $T_{R}$ are independent of $R$ for $R \gtrsim L$, our approximation is also expected to be exact for $R$ in that range. In conclusion, replacing the $\boldsymbol{R}$ pdf by a delta function at the rms distance is quite well justified. It may overestimate $S_{\text {Tij }}$ and $\mathcal{D}_{i j}$ by $\sim 20 \%$, or the relative speed by $\sim 10 \%$, if the primary distance is in the inertial range (which is the case for $\tau_{p}$ in the inertial range).

\section{Results}

To calculate the radial relative velocity, we need the longitudinal particle structure function, $S_{\text {pll }}$, which in turn requires $\mathcal{A}_{l l}$ and $\mathcal{D}_{l l}$. From eq. (2.19), it is clear that $\mathcal{A}_{l l}=$ $\mathcal{A}_{n n}$ and

$$
\mathcal{A}_{l l}=\frac{\left(\Omega_{2}-\Omega_{1}\right)^{2}\left(\Omega_{1} \Omega_{2}+\left(\Omega_{1}+\Omega_{2}\right) \frac{z^{2}}{2}\right)}{\left(\Omega_{1}+\Omega_{2}\right)\left(\Omega_{1}+\Omega_{1}^{2}+\frac{z^{2}}{2}\right)\left(\Omega_{2}+\Omega_{2}^{2}+\frac{z^{2}}{2}\right)} u^{\prime 2} .
$$

We obtain $\mathcal{D}_{l l}$ using the relation $\mathcal{D}_{l l}=\mathcal{D}_{i j} r_{i} r_{j} / r^{2}$. From eq. (2.38) for $\mathcal{D}_{i j}$, we have

$$
\begin{aligned}
\mathcal{D}_{l l}(r)=\int_{-\infty}^{0} \frac{d \tau}{\tau_{p 1}} \int_{-\infty}^{0} & \frac{d \tau^{\prime}}{\tau_{p 2}}\left[\left(\frac{1}{3}+\frac{2 r^{2}}{3 R^{2}}\right) S_{l l}(R)+\left(\frac{2}{3}-\frac{2 r^{2}}{3 R^{2}}\right) S_{n n}(R)\right] \\
& \times \Phi\left(\tau-\tau^{\prime} ; \tau_{T R}(R), T_{R}(R)\right) \exp \left(\frac{\tau}{\tau_{p 1}}\right) \exp \left(\frac{\tau^{\prime}}{\tau_{p 2}}\right) .
\end{aligned}
$$

We will need the 3D relative velocity under the assumption of ballistic particle separation. In that case, we take the $3 \mathrm{D}$ relative velocity variance, $\left\langle w^{2}\right\rangle$, to be $S_{\mathrm{p} i i}=\mathcal{A}_{i i}+\mathcal{D}_{i i}$ (see §2.2). Using contractions of eqs. (2.19) and (2.38), we have,

$$
\left\langle w^{2}\right\rangle=3 \mathcal{A}_{l l}+\int_{-\infty}^{0} \frac{d \tau}{\tau_{p 1}} \int_{-\infty}^{0} \frac{d \tau^{\prime}}{\tau_{p 2}}\left(S_{l l}(R)+2 S_{n n}(R)\right) \Phi\left(\tau-\tau^{\prime} ; \tau_{T R}, T_{R}\right) \exp \left(\frac{\tau}{\tau_{p 1}}\right) \exp \left(\frac{\tau^{\prime}}{\tau_{p 2}}\right)
$$

where $\mathcal{A}_{l l}$ is given by eq. (3.1).

\subsection{The Monodisperse Case}

As discussed earlier, for identical particles with $\tau_{p 1}=\tau_{p 2}=\tau_{p}, \mathcal{A}_{i j}$ vanishes and only $\mathcal{D}_{i j}$ contributes to the particle velocity structure tensor, i.e., $S_{\mathrm{p} i j}=\mathcal{D}_{i j}$. Thus the longitudinal particle velocity structure function is given by,

$$
\begin{aligned}
S_{\mathrm{p} l l}(r)=\int_{-\infty}^{0} \frac{d \tau}{\tau_{p}} \int_{-\infty}^{0} & \frac{d \tau^{\prime}}{\tau_{p}}\left[\left(\frac{1}{3}+\frac{2 r^{2}}{3 R^{2}}\right) S_{l l}(R)+\left(\frac{2}{3}-\frac{2 r^{2}}{3 R^{2}}\right) S_{n n}(R)\right] \\
& \times \Phi\left(\tau-\tau^{\prime} ; \tau_{T R}(R), T_{R}(R)\right) \exp \left(\frac{\tau}{\tau_{p}}\right) \exp \left(\frac{\tau^{\prime}}{\tau_{p}}\right) .
\end{aligned}
$$

To solve this equation, one needs the particle dispersion laws for $\rho$ to calculate the distance $R$ by eq. (2.24). We start with the two simplified cases in order to study the effect of each phase in the two-phase separation found by Bec et al. (2009a). 

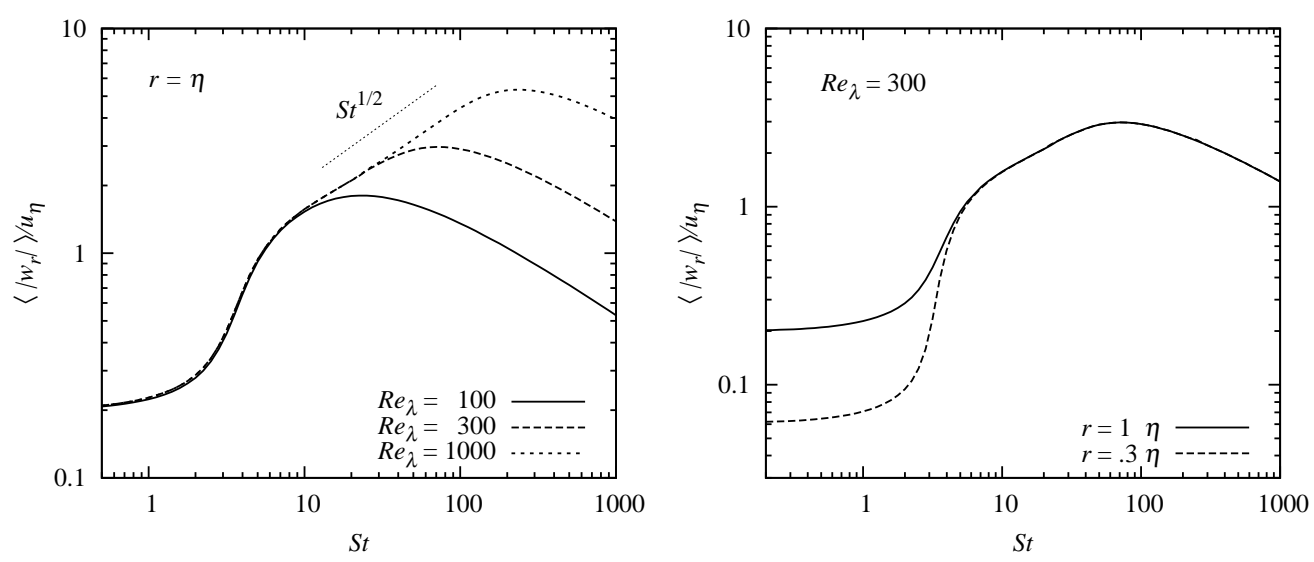

FiguRE 1. The radial relative velocity, $\left\langle\left|w_{r}\right|\right\rangle$, as function of the Stokes number, St, for ballistic particle separation. The left panel shows results for different $R e_{\lambda}$. The distance $r$ is fixed at $\eta$. A $S t^{1 / 2}$ scaling is found between the S-T limit and the large $S t$ limit. The Stokes number at the transition from $S t^{1 / 2}$ to $S t^{-1 / 2}$ scalings increases linearly with $R e_{\lambda}$. The right Panel shows the dependence on $r$ with $R e_{\lambda}$ set to 300 .

\subsubsection{Ballistic Separation Behavior}

We first consider the effect of the ballistic separation phase by using eq. (2.35) for the particle distance at all times. Eq. (3.3) gives the variance of the 3D relative velocity, $\left\langle w^{2}\right\rangle$, needed in eq. (2.35). For identical particles, eq. (3.3) becomes,

$$
\left\langle w^{2}\right\rangle=\int_{-\infty}^{0} \frac{d \tau}{\tau_{p}} \int_{-\infty}^{0} \frac{d \tau^{\prime}}{\tau_{p}}\left(S_{l l}(R)+2 S_{n n}(R)\right) \Phi\left(\tau-\tau^{\prime} ; \tau_{T R}, T_{R}\right) \exp \left(\frac{\tau}{\tau_{p}}\right) \exp \left(\frac{\tau^{\prime}}{\tau_{p}}\right) .
$$

Because the distance $R$ in the integral on the r.h.s. depends on $\left\langle w^{2}\right\rangle$, eq. (3.5) is implicit for $\left\langle w^{2}\right\rangle$. We numerically solve eq. (3.5) by an iterative method. After obtaining $\left\langle w^{2}\right\rangle$, we use it to calculate $S_{\mathrm{p} l l}$ from eq. (3.4).

The results for the radial relative velocity, $\left\langle\left|w_{r}\right|\right\rangle$, as a function of the Stokes number, St, are shown in Fig. 1. We obtained $\left\langle\left|w_{r}\right|\right\rangle$ from the conversion $\left\langle\left|w_{r}\right|\right\rangle=\sqrt{2 S_{\mathrm{pll}} / \pi}$ assuming a Gaussian distribution for $w_{r}$. We simply follow this convention here although we realize that the relative velocity distribution is broader than Gaussian for small particles in high Reynolds-number flows (e.g., Sundaram \& Collins (1997); Wang et al. (2000), see also Bec et al. (2009b) for the intermittency in inertial particle structures). We will give the relative speed across fixed distances as a function of Stokes numbers (for convenience in the comparison with simulations in $§ 3.1 .3$ ), although in coagulation models one needs to use the relative speed across the particle size as a function of the size. The latter can be easily calculated from our model with the Stokes number as a function of the particle size in a specific application.

In the left panel of Fig. 1, we plot $\left\langle\left|w_{r}\right|\right\rangle$ for particles at a distance $r=\eta$ as a function of the Stokes number and the Taylor Reynolds number. The solution reproduces the S-T limit (with the relative speed independent of $S t$ ) and the $S t^{-1 / 2}$ scaling in the limit of large friction time. As in the model by Zaichik et al., the relative velocity is found to scale as $S t^{1 / 2}$ for intermediate Stokes numbers. This scaling corresponds to the inertial-range scaling of the turbulent flow and the $S t^{1 / 2}$ scaling range will be referred to as the inertial range.

The $S t^{1 / 2}$ scaling can be understood as follows. As discussed earlier, the main contribu- 
tion to the integrals in eqs. (3.4) and (3.5) is from $\tau^{\prime}, \tau \sim-\tau_{p}$, and the particle distance, $R$, at $\tau, \tau^{\prime} \sim-\tau_{p}$, called the primary distance in $\S 2.3 .3$, is important in determining the relative velocity. We will denote the primary distance as $R_{p}$. The primary distance as a function of $\tau_{p}$ is evaluated by $R_{p}=R\left(-\tau_{p},-\tau_{p}\right)$ using eq. (2.22). For intermediate Stokes numbers, $R_{p}$ is much larger than $r$, we thus have $S_{\mathrm{p} l l}=\left\langle w^{2}\right\rangle / 3$ from eqs. (3.4) and (3.5). Thus the scaling behavior of $\left\langle w_{r}^{2}\right\rangle$ with $\tau_{p}$ is the same as that of $\left\langle w^{2}\right\rangle$. The latter can be obtained by analyzing eq. (3.5).

The $\Phi$ term in eq. (3.5) represents the persistency of a structure of size $R$. It is approximately given by $\exp \left(-\left|\tau-\tau^{\prime}\right| / T_{R}(R)\right)$. The effect of this factor depends on how $T_{R}\left(R_{p}\right)$ at the primary distance, $R_{p}$, compares to $\tau_{p}$. If $T_{R}\left(R_{p}\right)$ is larger than $\tau_{p}, \Phi$ would be essentially unity for $\tau$ and $\tau^{\prime}$ values that significantly contribute to the integral in eq. (3.5). One the other hand, if $T_{R}$ at $R_{p}$ is smaller than $\tau_{p}$, a factor of $T_{R}\left(R_{p}\right) / \tau_{p}$ needs to be accounted because the $\Phi$ factor suggests that, for a given $\tau$, only $\tau^{\prime}$ that satisfies $\left|\tau^{\prime}-\tau\right| \lesssim T_{R}$ (instead of the range $-\tau_{p} \lesssim \tau^{\prime} \leqslant 0$ ) contributes significantly to the integral. We find the latter is the case from our numerical solution. Thus, considering the main contribution to the integral is from the integrand at $R \sim R_{p}$, we expect $\left\langle w^{2}\right\rangle \propto\left[S_{l l}\left(R_{p}\right)+2 S_{n n}\left(R_{p}\right)\right] T_{R}\left(R_{p}\right) / \tau_{p}$. For ballistic separation, the primary distance $R_{p} \simeq\left\langle w^{2}\right\rangle^{1 / 2} \tau_{p}$. And for $R$ in the inertial range, we have $S_{l l}(R), S_{n n}(R) \propto R^{2 / 3}$ and $T_{R}(R) \propto R^{2 / 3}$ (eqs. (2.28), (2.32)). These scalings give $\left\langle w^{2}\right\rangle \propto\left\langle w^{2}\right\rangle^{2 / 3} \tau_{p}^{1 / 3}$, which results in $\left\langle w^{2}\right\rangle \propto \tau_{p}$ and hence the $S t^{1 / 2}$ scaling for the relative velocity.

As the Stokes number increases, the relative speed reaches a peak, and transitions to the $S t^{-1 / 2}$ scaling. This change occurs when $R_{p} \simeq L$ and corresponds to the switch of the scaling behaviors of $S_{l l}, S_{n n}$, and $T_{R}$ from the inertial range to the outer scales. As pointed out earlier, if the primary distance, $R_{p}$, is much larger than $L$, the particle velocities are uncorrelated. A $S t^{-1 / 2}$ scaling is expected from the particle velocity variances, eq. (2.21). This scaling can also be obtained using an analysis similar to that for the $S t^{1 / 2}$ scaling given above. If $R_{p} \gg L$, the structure functions and the timescale $T_{R}$ that give the primary contribution to the integrals in eqs. (3.4) and (3.5) are constant, i.e., $S_{l l}=S_{n n}=$ $2 u^{\prime 2}$ and $T_{R}=T_{L}$ (eqs. (2.29) and (2.33)). Therefore, eq. (3.5) gives $\left\langle w^{2}\right\rangle \propto u^{\prime 2} T_{L} / \tau_{p}$, and hence the $S t^{-1 / 2}$ scaling. Again the factor $T_{L} / \tau_{p}$ comes from the $\Phi$ term.

The Stokes number, $S t_{m}$, or the friction time, $\tau_{p m}$, at which the relative speed reaches the maximum can be approximately obtained by setting $R_{p} \sim L$. Using $R_{p} \simeq\left\langle w^{2}\right\rangle^{1 / 2} \tau_{p}$ for the ballistic separation and $\left\langle w^{2}\right\rangle^{1 / 2} \simeq 1.0 S t^{1 / 2} u_{\eta}$ in the inertial range from our numerical solution, we find that $S t_{m} \simeq R e_{\lambda} / \sqrt{15}$, or $\tau_{p m} \simeq T_{e}$, where $T_{e}$ is defined as $u^{\prime 2} / \bar{\epsilon}$. The order-of-magnitude estimate for $S t_{m}$ turns out to be in good quantitative agreement with the numerical solution in the left panel of Fig. 1, which also confirms the linear increase of $S t_{m}$ with the $R e_{\lambda}$.

The right panel of Fig. 1 shows the dependence of the relative velocity on $r$, the particle distance at time zero. For $r \lesssim \eta$ of interest here, the relative velocity depends on $r$ only in the S-T limit where it increases linearly with $r$ (see the shear term in eq. (1.1)). For larger particles, it becomes independent of $r$. This is because in that case the contribution is mainly from the particle memory of the flow velocity difference when the particle distance was much larger than $r$.

We have used the same distance, $R$, for the timescale $T_{R}$ in the $\Phi$ term as that for the structure functions. As discussed in $\S 2.2$, for $T_{R}$ it might be a better choice to use the particle distance, $R_{m}$, at the earlier one $\left(\min \left(\tau, \tau^{\prime}\right)\right)$ of the two times $\tau$ and $\tau^{\prime}$. With this choice, $T_{R}$ is larger because the separation is larger at earlier time. The predicted relative velocity is also larger, and we find an increase by $\sim 30 \%$ in the inertial range. 

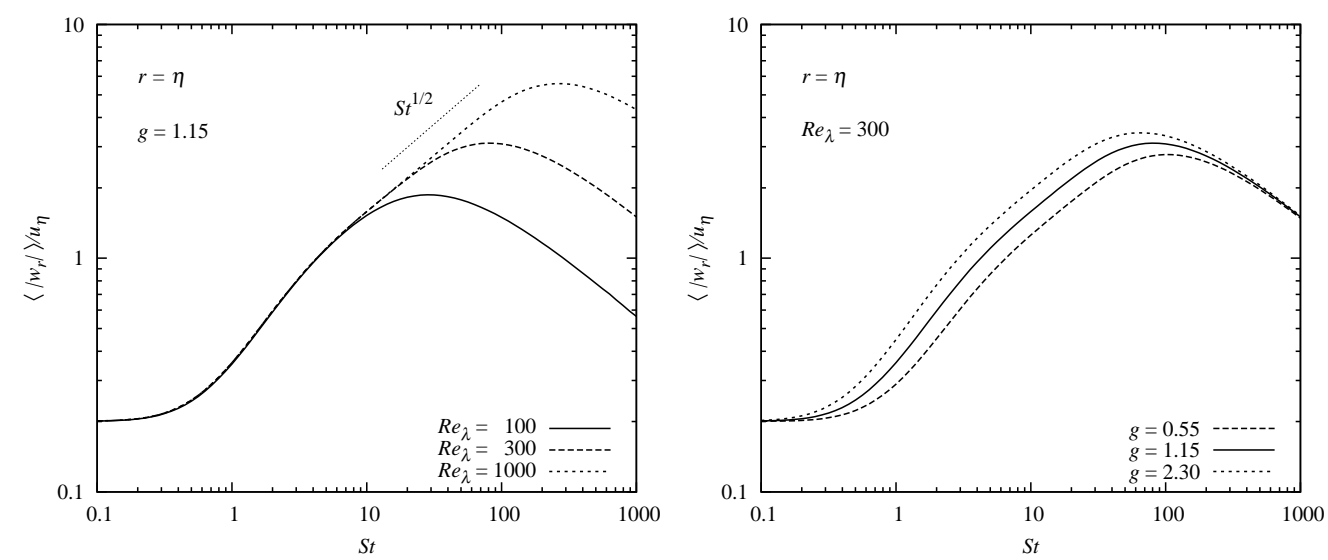

FiguRE 2. The radial relative velocity, $\left\langle\left|w_{r}\right|\right\rangle$, at $r=\eta$ as a function of the Stokes number, St, assuming the R-O separation followed by a diffusive phase. The left panel shows results for different $R e_{\lambda}$. As in the case of ballistic separation, a $S t^{1 / 2}$ scaling is found in the inertial range. The Stokes number where the curve peaks also increases linearly with $R e_{\lambda}$. The right panel gives the dependence of the relative velocity on $g$. In the inertial range it increases with $g$ as $g^{1 / 3}$.

\subsubsection{Tracer-like Separation Behavior}

In this subsection, we examine the effect of the tracer-like separation phase. Our main purpose here is to study how the relative velocity scales with the Stokes number from the R-O law.

Here we will consider an initial distance of $r=\eta$, and start the separation with $\rho(\tau)^{2}=r^{2}+g \bar{\epsilon}|\tau|^{3}$. We switch to the diffusive regime when the separation exceeds $L$ (§2.3), and the connection is chosen such that $\rho(\tau)^{2}=L^{2}+2 D\left|\tau-\tau_{d}\right|$ for $|\tau|>\left|\tau_{d}\right|$, where $\tau_{d}$ is the time when $\rho$ reaches $L$. In Fig. 2, we show the results for the relative velocity as a function of $S t$ and $R e_{\lambda}$ for particles at $r=\eta$. The Richardson constant is set to be $g=1.15$. The solution correctly reproduces the limits at small and large friction timescales as expected, and interestingly, we also find a $S t^{1 / 2}$ scaling for the relative velocity in the intermediate range of $S t$.

To explain the $S t^{1 / 2}$ scaling, we analyze again $S_{\text {pll }}$ by considering the integrand in eq. (3.4) at the primary distance, $R_{p}$. For $R_{p} \gg r$, eq. (3.4) can be approximately written as $S_{\mathrm{p} l l} \sim\left[S_{l l}\left(R_{p}\right)+2 S_{n n}\left(R_{p}\right)\right] T_{R}\left(R_{p}\right) /\left(3 \tau_{p}\right)$. The factor $T_{R}\left(R\left(\tau_{p}\right)\right) / \tau_{p}$ (which is smaller than 1 for any $g \lesssim 10)$ is based on the same reasoning as in $\$ 3.1 .1$ for the ballistic case. With the R-O separation law, the primary distance is given by $R_{p} \simeq\left(g \bar{\epsilon} \tau_{p}^{3}\right)^{1 / 2}$. Therefore, using the inertial-range scalings of $S_{l l}, S_{n n}$, and $T_{R}$, we have $S_{\mathrm{p} l l} \propto g^{2 / 3} \tau_{p}$. This explains the $S t^{1 / 2}$ scaling for the radial relative velocity in the inertial range, and also predicts that the relative velocity increases with the Richardson constant as $g^{1 / 3}$. The $g^{1 / 3}$ dependence is confirmed by the right panel of Fig. 2. The increase of the relative velocity with $g$ shows that faster particle separation gives larger relative velocity.

As in the ballistic case, the friction time, $\tau_{p m}$, at which the relative velocity peaks, is again obtained by setting the primary distance $R_{p}$ to $L$. Using the R-O separation law for $R_{p}$, we find that $\tau_{p m} \simeq g^{-1 / 3} T_{e}$, with $T_{e}=u^{\prime 2} / \bar{\epsilon}$. In units of the Kolmogorov timescale, we have $S t_{m} \simeq g^{-1 / 3} R e_{\lambda} / \sqrt{15}$. This is consistent with the results in the left panel of Fig. 2.

We have finished the study for the effects of each separation phase in the two-phase separation found by Bec et al. (2009a). We found that, in the inertial range, the relative 
speed from using the R-O law has the same scaling behavior as from the ballistic separation. Thus the same $S t^{1 / 2}$ scaling is also expected for a combination of ballistic and R-O separation behaviors. For $g \sim 1$, the predicted value for the relative speed in the inertial range from the R-O separation is quantitatively very close to that in the ballistic case, and so is the peak Stokes number $S t_{m}$. Therefore for the combined behavior the prediction for the inertial range and for the switch to large Stokes numbers would be similar to the two simplified cases (see Fig. 4). However, in the transition region from the S-T limit to inertial range, the predicted relative speed is quite different for the two separation behaviors.

\subsubsection{Combined Separation Behavior and Comparison with Simulation Results}

We use the simulation results by Wang et al. (2000) to test our model. The Reynolds numbers in their simulations are quite low with $R e_{\lambda}$ in the range from 45 to 75 . The results by Wang et al. (2000) for three different Reynolds numbers are shown as data points in Fig. 3. The relative speed is for particles at a separation of $\eta$. Due to the limited resolution of these simulations, no inertial-range scaling is seen. For $R e_{\lambda}$ in the same range, our model with the two separation behaviors considered above does not show the $S t^{1 / 2}$ scaling either (see $R e_{\lambda}=100$ curves in Figs. 1 and 2).

The separation behavior of inertial particle pairs at Reynolds numbers as those in the simulations by Wang et al. (2000) is unknown. We tried different separation behaviors and compared the predictions for the relative speed with their results. We find that a combination of an early ballistic phase and a later tracer-like phase, as found by Bec et al. (2009a), can give a quite good fit to the results by Wang et al. (2000) (while with a pure ballistic separation or a pure tracer-like separation no satisfactory fit is found). The lines in Fig. 3 show the predicted relative velocities with such a two-phase separation, which agree quite well with the simulation data. The exact separation behavior used in Fig. (3) is as follows. The separation starts with a ballistic phase which lasts from time zero back to $\tau_{c}$. At $\tau_{c}$, it continuously connects to the R-O separation law, and finally switches to the diffusive regime when the separation exceeds $L$. The connection between the ballistic phase (eq. (2.35)) and the R-O phase (eq. 2.36) is chosen such that the particle distance at $\tau<\tau_{c}$ is given by $\rho(\tau)^{2}=\rho\left(\tau_{c}\right)^{2}+g\left|\tau-\tau_{c}\right| \tau^{2}$. This connection between the two phases is quite smooth. Furthermore, we set $\tau_{c}=-1.4 \tau_{p}$ and $g=1$. The connection between the $\mathrm{R}-\mathrm{O}$ regime and the diffusive regime is the same as that for the tracer-like separation behavior discussed in §3.1.2. Due to the low Reynolds numbers here, there is only a very short period for the $\mathrm{R}-\mathrm{O}$ separation between the ballistic regime and the diffusive regime in the chosen separation behavior. How the ballistic phase exactly connects to the R-O phase in the backward dispersion of inertial particles is unknown. We also tried other ways to connect the two phases, and found that, by adjusting $\tau_{c}$ and $g$, some other connections can also give satisfactory fits. For example, if the two phases are connected by $\rho(\tau)^{2}=\rho\left(\tau_{c}\right)^{2}+g\left(\tau-\tau_{c}\right)^{2}|\tau|$ for $\tau<\tau_{c}$, fitting the simulation data gives $\tau_{c}=-1.1 \tau_{p}$ and $g=1.5$; and for a connection with $\rho(\tau)^{2}=\rho\left(\tau_{c}\right)^{2}+g|\tau|^{3}-g\left|\tau_{c}\right|^{3}$ in the R-O phase, we find a good fit with $\tau_{c}=1.5 \tau_{p}$ and $g=0.6$.

Because $\mathcal{D}_{i j}$ is an integral over the history of the particle distance, different separations as a function of time could lead to the same predicted relative speed. Therefore, the exact backward dispersion behavior cannot be determined by fitting the simulation results for the relative speed. In other worlds, a separation law that fits the data may not represent the exact dispersion behavior for inertial particles in the simulated flows by Wang et al. (2000), and a verification would need a direct numerical study of the pair separation. However, the fact that the dispersion behavior used in Fig. 3 is generally consistent with the simulations results by Bec et al. (2009a) (see their Fig.8 where the connection 


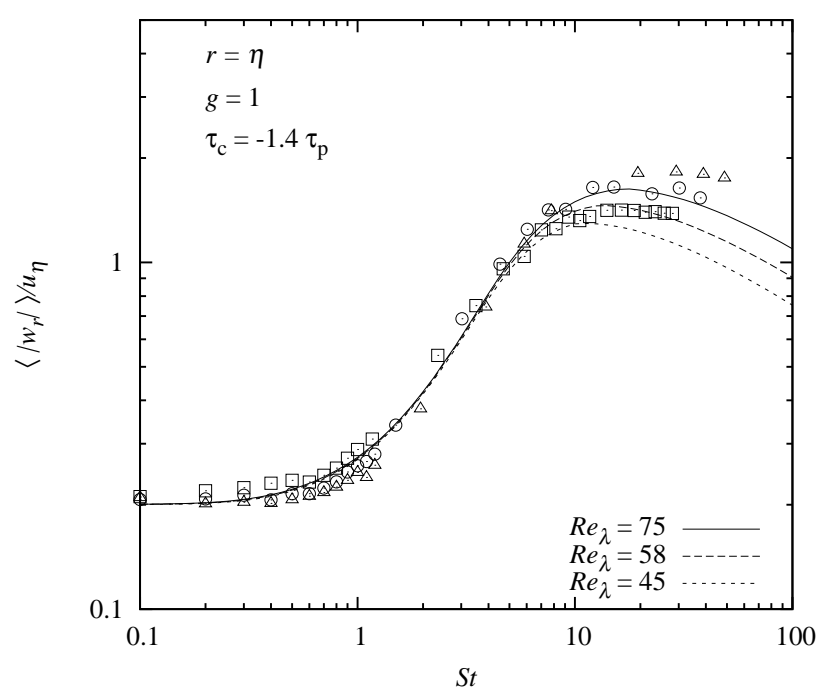

Figure 3. Comparison with the simulation results from Wang et al. $(2000)$ at $R e_{\lambda}=45$ (squares), 58 (circles) and 75 (triangles). Lines are the predicted relative speed from our model with a two-phase separation behavior, a ballistic phase followed a tracer-like phase. The ballistic separation is assumed to connect with the R-O separation with $g=1$ at $-1.4 \tau_{\mathrm{p}}$. See text for details on this connection.

between the ballistic phase and the R-O phase occurs between 1-2 $\tau_{p}$ ) suggests that the adopted behavior is at least qualitatively correct. We will adopt the separation behavior used in Fig. 3 for all the calculations in the rest of the paper.

We find a significant deviation ( up to $25 \%$ ) between the model prediction and the simulation results for very large particles $(S t \gtrsim 20)$. It seems that the deviation at these large Stokes numbers could not be removed by a reasonable change in the separation behavior without causing discrepancy at smaller St. This deviation also occurs in Zaichik et al.'s model. An immediate suspect for this deviation is the assumption in our model (and in Zaichik et al.'s model) that the trajectories of all particles are not far away from those of the fluid elements This assumption is not well justified for very large particles. As discussed at the end of $\S 2.2$, the temporal correlation of the flow velocity (or the velocity difference) along the trajectories of large particles may be close to the Eulerian correlation, while we used the Lagrangian correlation timescale throughout the model. If the Eulerian correlation timescale were used for these particles, and if the Eulerian correlation timescale is larger than the Lagrangian timescale (see discussions in $\$ 2.2$ ), the predicted relative speed for large particles would be larger, reducing the the difference between the model and the simulation results. Our approximation for the trajectory structure tensor in $\S 2.2$ could also contribute to the discrepancy.

Using the separation behavior in Fig. 3 that well fits the simulation results, we carried out calculations for larger Reynolds numbers. Fig. 4 shows our prediction for $R e_{\lambda}=300$. For comparison, we included results assuming pure ballistic separation (the dashed thin line) and pure tracer-like separation (the solid thin line). For the latter we used $g=1$ for the R-O separation. We see that, in the two-phase case, the relative velocity in the transition region from the S-T limit to the inertial range lies between the two cases with single separation behavior. In this transition region, the predicted relative speed relies on how the ballistic phase and the R-O phase exactly connects. In the inertial range, we again have the $S t^{1 / 2}$ scaling, which is expected because both the ballistic behavior and 


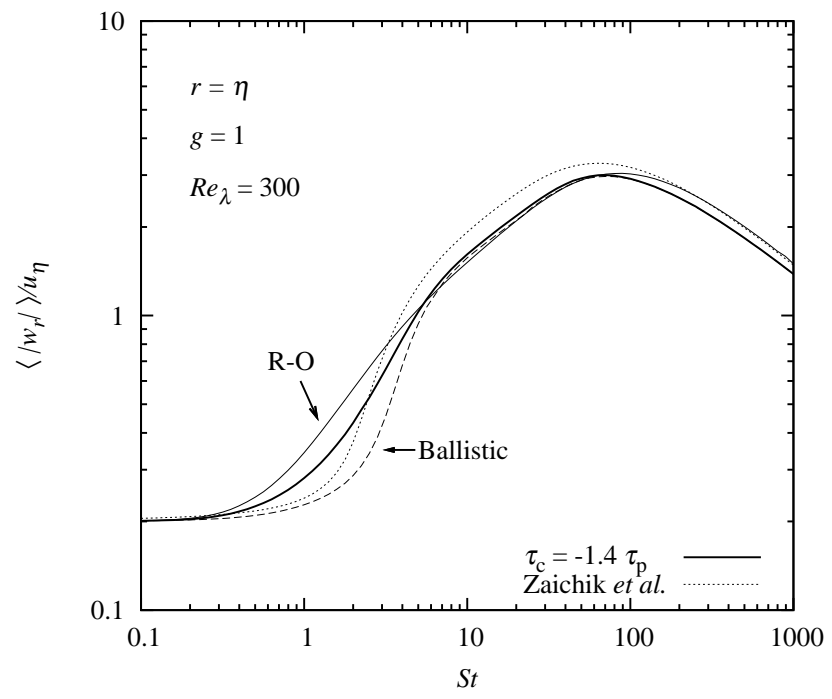

FigURE 4. The radial relative velocity, $\left\langle\left|w_{r}\right|\right\rangle$, as a function of the Stokes number, $S t$, with the same two-phase separation used in Fig. 3. The dashed thin line is for ballistic separation only and the solid thin line is for tracer-like behavior only (with $g=1$ for the R-O separation). Also shown is the prediction by the Zaichik et al.'s model.

the R-O separation law give that scaling. For $g \sim 1$, the predicted relative speed turns out to be very similar for all the three cases in the inertial range. The predicted speed in the inertial range is not significantly affected by the details of the connection between the ballistic and the R-O phases. The Stokes number at the transition from the inertial range to the $S t^{-1 / 2}$ range is $\sim R e_{\lambda} / \sqrt{15}\left(\tau_{p} \sim T_{e}\right)$, similar to both the pure ballistic case and the pure tracer-like case.

Fig. 4 also shows the relative speed predicted by Zaichik et al.'s model (the thin dotted line). We obtained the results of their model by numerically solving the set of differential equations given in Zaichik et al. (2006) (i.e., their eqs. (51-53)). The transition from the S-T limit to the inertial range in Zaichik et al.'s model is quite steep. The relative speed in the inertial range in their model is $20 \%$ larger than the our model. This is probably because a faster particle separation is built into Zaichik et al.'s model. As pointed our earlier, in the limit of $\tau_{p} \rightarrow 0$, Zaichik et al.'s formulation implies a Richardson constant significantly larger than 1 .

\subsection{The Bidisperse Case}

We first point out the importance of gravity in the bidisperse case. In the monodisperse case, the settling velocity by gravity is the same for all particles, and thus neglecting gravity in that case may give approximately good estimates for the relative speed. The situation is quite different in the bidisperse case where gravity may play a major role, especially for large particles. The terminal velocity difference between two different particle could give substantial or even dominant contribution to their relative speed. Besides the direct contribution to the relative speed, differential settling can also have an indirect effect by increasing the particle separation in the past. Since a larger separation backward in time would increases the contribution from the particle memory of the flow velocity difference, this indirect effect also tends to give a larger collision speed.

We will neglect gravity in our model below. Clearly, this limits the application of the model only to situations where the relative speed caused by differential settling is 


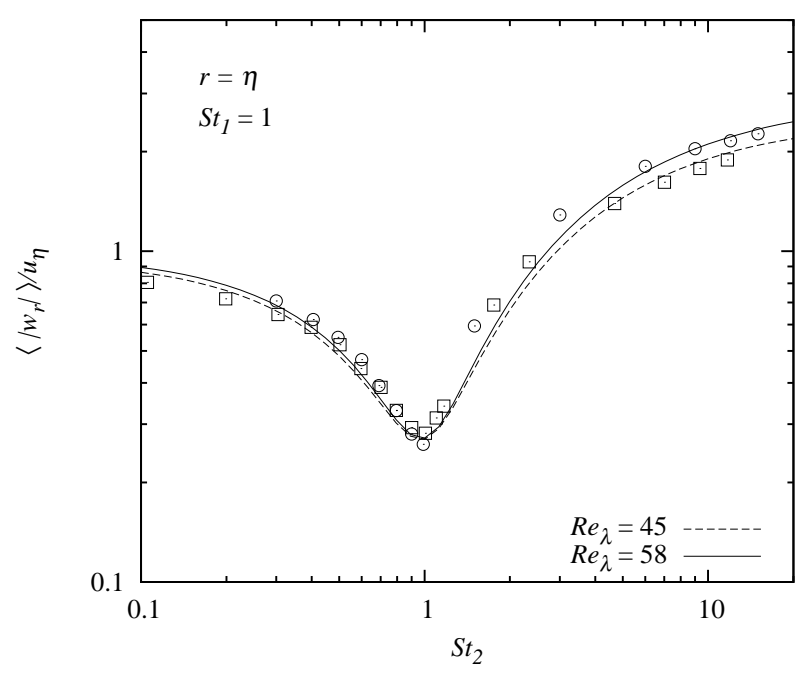

FiguRE 5. Comparison with the simulation results from Zhou et al. $(2001)$ at $R e_{\lambda}=45$ (squares), 58 (circles). Lines are the predicted relative speed from our model with a two-phase separation behavior, a ballistic separation followed by a tracer-like behavior. The ballistic separation is assumed to connect with the $\mathrm{R}-\mathrm{O}$ separation with $g=1$ at $-1.4 \tau_{\mathrm{p}}$. See text for details about the connection.

negligible in comparison to the prediction of our model. However, understanding the simpler case with turbulence alone is of theoretical importance because it serves as the first step to a physical and accurate model for particle collisions in realistic environments where both turbulence and gravity are present. The effect of gravity may be included in our framework by accounting for both the direct effect of differential settling and its indirect effect through the backward separation of the particles (e.g., in a similar way as in Avala et al. (2008), who, however, did not include the particle separation by the turbulent flow).

In the bidisperse case, we include the contribution from the $\mathcal{A}_{l l}$ term, eq. (3.1), to the relative speed. We use the same numerical method to solve $\mathcal{D}_{l l}$ as in the monodisperse case. The particle separation behavior is chosen to be similar to the one that well fits the simulation results in the monodisperse case. Note that in the ballistic phase, the separation speed (i.e., the relative velocity variance, $\left\langle w^{2}\right\rangle$ ) to be used for the calculation of $\mathcal{D}_{l l}$ has a contribution from the $\mathcal{A}_{i i}$ term (eq. 3.3). It is not clear how long the ballistic phase lasts in the bidisperse case, since the friction timescales of the two particles are different. We simply assume that the duration of the ballistic separation is proportional to the average of the two friction timescales. Fig. 5 shows the radial relative velocity as a function of the Stokes number of particle (2) for a fixed Stokes number, $S t_{1}=1$, of particle (1). The data points are simulation results from Zhou et al. (2001) for $r=\eta$ at $R e_{\lambda}=45$ and 58. These data are from Fig. 15 of Zhou et al. (2001), but a different normalization is used here. The lines are the prediction of our model where we adopted the same connection used in Fig. 3 for the monodisperse case and set $g=1$ and $\tau_{\mathrm{c}}=$ $-1.4 \times\left(\tau_{\mathrm{p} 1}+\tau_{\mathrm{p} 2}\right) / 2$. The agreement of the model prediction with the simulation results is quite good, except that it is a little bit broader around the dip. A possible reason is that the separation behavior for different particles is different from the one used here, which is based on the separation behavior of identical particle pairs.

We give more details for the relative speed between different particles in Fig. 6, where 


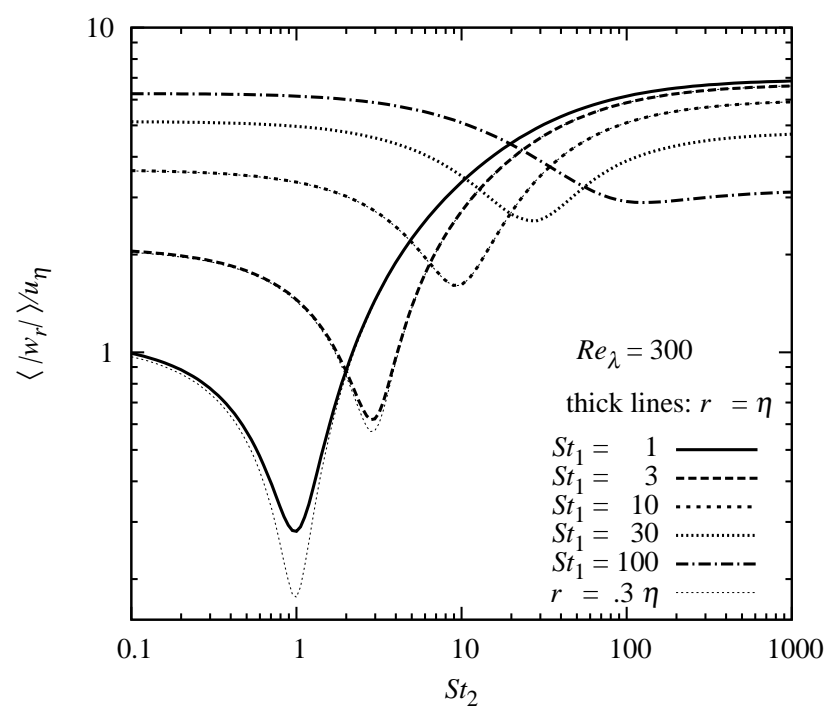

FIgURE 6 . The radial relative velocity, $\left\langle\left|w_{r}\right|\right\rangle$ as a function of $S t_{2}$ with fixed $S t_{1}$. The thick lines give the relative speed at $r=\eta$. Each curve has a dip at $S t_{2}=S t_{1}$. The three thin dotted lines show the results for $r=0.3 \eta$ with $S t=1,3$, and 10 .

the Taylor Reynolds number is set to 300. The thick lines are for $r=\eta$. A dip around $S t_{2} \sim S t_{1}$ is found in every curve with $S t_{1} \lesssim 100$. The existence of the dips is related to the fact that the contribution from $\mathcal{A}_{i j}$ for particles of similar sizes is small. Physically, it means that the velocities of similar particles tend to have stronger correlation than particles with very different friction timescales. Around each dip, the contribution to the relative velocity is mainly from $\mathcal{D}_{l l}$, while far from the dip it is dominated by $\mathcal{A}_{l l}$. A comparison of the two terms shows that they give similar contributions when the Stokes number ratio is about 3-4. If the friction timescales of the two particles differ by a factor much larger than 4, using the generalized acceleration term alone may give a satisfactory result. The $\mathcal{D}_{l l}$ and $\mathcal{A}_{l l}$ terms in our model are closely related to the two terms in the equation for the velocity difference given in Bec et al. (2005) (their equation (13)). Their discussion on the relative importance of those two terms provides physical insights to understand the dips in Fig. 6. The relative speeds at the dip centers correspond to equal Stokes numbers. Connecting these centers would give a curve identical to that for the monodisperse case with the same separation behavior and parameters.

On the far left of the dip, the relative velocity approaches a constant. The constant corresponds to $\mathcal{A}_{l l}$ in the limit $\Omega_{2} \rightarrow 0$, which, from eq. (3.1), is given by $\frac{\Omega_{1}^{2}}{\Omega_{1}+\Omega_{1}^{2}+z^{2} / 2} u^{\prime 2}$. As $S t_{1}$ (or $\Omega_{1}$ ) increases, the dip moves to the right, and the relative velocity on the far left increases. It reaches and stays at the maximum (corresponding to $\mathcal{A}_{l l}=u^{\prime 2}$ ) after $\Omega_{1}$ becomes much larger than 1 . The opposite occurs on the far right of the dip, i.e., in the limit $S t_{2} \gg S t_{1}$. In this limit, $\mathcal{A}_{l l}$ approaches $\frac{\Omega_{1}+z^{2} / 2}{\Omega_{1}+\Omega_{1}^{2}+z^{2} / 2} u^{\prime 2}$, which has a maximum of $u^{\prime 2}$ at small $S t_{1}$. With increasing $S t_{1}$ or $\Omega_{1}$, the relative velocity decreases on the far right of the dip, while it increases on the other side.

The dip disappears for very large $S t_{1}$ (larger than $\sim 100$ for the case shown in the Fig. 6). This can be explained as follows. Physically, the dips are due to strong velocity correlation between particles of similar sizes. Thus no dip would exist if the friction time of particle (1) is such that its velocity is not significantly correlated with any particle of 
similar size. This is the case for a particle with $\tau_{p}$ larger than $\tau_{p m}$, the friction timescale where the relative speed peaks in the monodisperse case (see §3.1). The velocity of such a particle is not correlated even with an identical particle. Therefore, no dip is expected around $S t_{2} \simeq S t_{1}$ if $\tau_{p 1} \gtrsim \tau_{p m} \simeq T_{e}$. Clearly, this argument suggests that the critical value of $S t_{1}$ where the dip starts to disappear is the same as the Stokes number where the relative speed peaks in the monodisperse case.

The dependence of the relative velocity on the distance $r$ for the bidisperse case is also illustrated in Fig. 6, where the dotted thin lines show results for $r=0.3 \eta$. The $r$ dependence only comes from $\mathcal{D}_{l l}$, because $\mathcal{A}_{l l}$ does not depend on $r$. Thus the dependence may exist only around dips where $\mathcal{D}_{l l}$ gives a significant contribution. As in the monodisperse case, $\mathcal{D}_{l l}$ is independent of $r$ (again for $r \lesssim \eta$ ) if the Stokes numbers of both particles are much larger than 1 . This suggests that, in the bidisperse case, the relative velocity is a function of $r$ only when $S t_{2} \simeq S t_{1} \lesssim 1$. In Fig. 6 we see that for $S t_{1}=1$ the depth of the dip increases with decreasing $r$, corresponding to the decrease of the relative velocity with $r$ in eq. (1.1) for small identical particles. The $r$-dependence is already weak for $S t_{1}=3$, and the relative velocity becomes completely independent of $r$ for $S t_{1}=10$.

In summary, we find that in the bidisperse case the dominant contribution to the relative velocity is from $\mathcal{D}_{l l}$ if the ratio of the two Stokes numbers is not larger than 3-4, or from $\mathcal{A}_{l l}$ if that ratio is larger. For a fixed Stokes number of one particle, $S t_{1}$, the relative velocity as a function of the Stokes number of the other particle, $S t_{2}$, shows a dip at $S t_{2} \sim S t_{1}$. The dip corresponds to a stronger velocity correlation between particles of similar sizes than between very different particles. The existence of a dip at equal Stokes numbers has consequences for the collision kernel (e.g., Bec et al. 2005; Zaichik et al. 2006).

\section{Comparison with Other Models}

Our model has already been compared with some previous models earlier in the paper. Here we discuss more models and present the comparisons more systematically.

\subsection{Volk et al. (1980)}

In the astrophysical literature, the relative velocities of dust grains, e.g., in the context of the coagulation growth of dust grains in protoplanetary disks, has almost always been estimated based on the model by Volk et al. (1980), and a later version of that model by Markiewicz et al. (1991). Here we discuss this model for the particle relative velocity and compare it with the physical picture presented in this paper.

Volk et al (1980) derived both the 1-particle rms velocity and the relative speed between two particles. Their model started by considering the effects of turbulent eddies of different sizes on a particle of a given friction timescale. They speculated that the effect of an eddy on the particle depends on the eddy size. The particle would basically move along with large eddies if the eddy turnover time is much larger than the particle friction timescale. On the other hand, the effect of eddies with turnover time much smaller than the friction time is argued to be like a "random kick" because the eddy would "die" within a friction timescale. Apparently "random kick" here only means that the particle does not "receive" the driving by these small eddies to a full extent. There is also another way that an eddy may behave like a random kick. Due to their inertia, particles have a different velocity from the flow. If the relative velocity between a particle and a turbulent eddy of a given size is such that the particle crosses and leaves the eddy within a friction timescale, then the particle would not receive a "full" kick from that eddy, and the effect of the eddy is a "random kick". Based on these considerations, Volk et al. defined a 
critical eddy size in Fourier space, $k^{*}$, such that the effect of eddies below this size is like random kicks.

In the model by Volk et al. (1980) for the rms velocity of a single particle, the wavenumber $k^{*}$ appears to be important because eddies of size smaller than the scale $l^{*}$, corresponding to $k^{*}$, are expected to be less efficient at "driving" particle motions than larger ones because the particle does not have chance to "fully" receive the energy from these eddies.

Volk et al.'s derivation for the relative speed between two particles did not consider the separation of two particles, which is essential in our physical picture. In their calculation for the relative speed, they continue to take $k^{*}$ as a crucial scale. They essentially assumed the velocities of two particles induced by eddies with wavenumber larger than $k^{*}$ are not correlated, and the contribution to the particle velocity correlation is only from larger eddies. This assumption is not justified.

We argue that it is unlikely that $k^{*}$ is crucial for the velocity correlation of two particles (although $k^{*}$ may be an important scale for the 1-particle rms velocity, as discussed above). Whether the particle relative motions induced by eddies at a given scale are correlated or not is probably determined by how the particle separation compares to the eddy size. If the distance of two particles is smaller than the size of an eddy they encounter, the particle motions induced by this eddy would be correlated even if the eddy size is smaller than $l^{*}$ (the contribution to the two particles from a same eddy should be correlated). In this case, Volk et al.'s assumption would underestimate the correlation and overestimate the relative velocity. On the other hand, contributions to the velocities of the two particles from eddies of size smaller than the particle distance would be uncorrelated, because each particle receives a contribution from a different eddy of that size and motions in different eddies are likely to be independent. Therefore, contrary to the assumption by Volk et al., particle motions induced by eddies of size larger than $l^{*}$ are not always correlated. They are independent if the size of these eddies is smaller than the particle separation. The argument above suggests that it is the particle separation (instead of $l^{*}$ or $k^{*}$ ) that determines the particle velocity correlation, and thus an explicit examination of the particle separation is required.

The model by Volk et al. for the relative speed for two particles may be interpreted as one that implicitly assumes that the typical particle separation, $R$, is around the scale $k^{*}$. However, this assumption cannot be physically justified since the definition of $k^{*}$ has nothing to do with the distance between two particles. Even if the value of $k^{*}$ turned out to be close the to typical distance between particles, it should probably be taken as a coincidence. Volk et al.'s model may be improved by incorporating the particle distance as a function of time within their formulation.

\subsection{Williams \& Crane (198:)}

Williams \& Crane (1983) started from the derivation of the relative velocities for particles in two limits: $\tau_{p} \ll T_{L}$ and $\tau_{p} \gg T_{L}$, and then obtained a "universal" formula by interpolation. In the limit of $\tau_{p} \ll T_{L}$, they assumed that the particle separation back in time can be neglected ( $(2.2)$, and found the relative velocity is given by eq. (2.20). As argued in $\S 2.2$, this result is not valid for similar particles especially for $S t \gg 1$. In the other limit of $\tau_{p} \gg T_{L}$, Williams and Crane (1983) considered a linear particle separation, and chose the separation rate assuming the particle velocities are not correlated in this limit.

Williams \& Crane (1983) found a "universal" formula that reproduces the results for two limits they considered. This formula is obtained from a mathematical interpolation, and thus does not incorporate the physics of the relative velocities between particles of 
intermediate inertia. We find that the formula gives a $S t^{3 / 2}$ scaling for identical particles with $\tau_{p} \lesssim T_{L}$, which is probably incorrect.

\subsection{Yuu (1984)}

Yuu (1984) derived a formula for the the relative velocity that consists of an acceleration term and a shear term. Neglecting the effect of the added mass term (which is negligible in gaseous flows) included in Yuu's calculations, the acceleration term is also given by eq. (2.20), i.e., exactly the same as Williams and Crane's result for the small particle limit. Yuu's shear term is much smaller than our generalized shear term, $\mathcal{D}_{i j}$, for intermediate to large particles, because his calculation did not consider the particle separation back in time, and thus the shear term does not account for particles' memory of the larger flow velocity difference they "saw" at earlier times. As pointed out in $\S .2 .2$, this leads to a significant underestimate of the contribution from $\mathcal{D}_{i j}$. As a consequence, Yuu's model is not valid for similar particles with $\tau_{p} \gtrsim \tau_{\eta}$.

\subsection{Kruis \& Kusters (1997)}

Kruis \& Kusters (1997) gave a generalization to the models of Williams \& Crane (1983) and Yuu (1984). They first noticed that Williams and Crane's result for particles with $\tau_{p} \ll T_{L}$ and Yuu's acceleration term (eq. (2.20)) do not reproduce that in eq. (1) for the S-T limit. Replacing the temporal energy spectrum in Williams \& Crane (1983) by one that incorporates the flow acceleration and corresponds to a temporal correlation function similar to our eq. (2.16), they were able to derive a formula for the $\tau_{p} \ll T_{L}$ case that correctly reduces to the acceleration term in the S-T limit. The formula is the same as our result for $\mathcal{A}_{i j}$ in the limit $z \ll 1$. Kruis \& Kusters (1997) also generalized the Williams and Crane model to include the added mass effect, which was considered in Yuu (1984). The effect is negligible in a gaseous flow, but may be important in liquid flows. Following Williams and Crane, a "universal" solution was obtained by interpolating the generalized results for the two limits. Therefore the Kruis and Kusters model shares the same weakness as Williams \& Crane (1983): the physical importance of the particle separation for the relative velocity of similar particles with $\tau_{p} \lesssim T_{L}$ is not included in the model, and the interpolated results for that case are probably incorrect.

\subsection{The analytical model of Zaichik et al. (2003, 2006)}

In addition to their differential model discussed earlier in details, Zaichik and collaborators also presented an analytical model. Assuming Gaussian statistics for both the flow and the particle velocities, the analytical model calculates the joint pdf of the velocities of two particles, $P\left(\boldsymbol{v}^{(1)}, \boldsymbol{v}^{(2)}\right)$, from the two-point joint pdf of particle and flow velocities, $P\left(\boldsymbol{v}^{(1)}, \boldsymbol{v}^{(2)}, \boldsymbol{u}^{(1)}, \boldsymbol{u}^{(2)}\right)$. Zaichik et al. (2003, 2006) approximated the latter by $P\left(\boldsymbol{v}^{(1)} \mid \boldsymbol{u}^{(1)}\right) P\left(\boldsymbol{v}^{(2)} \mid \boldsymbol{u}^{(2)}\right) P\left(\boldsymbol{u}^{(1)}, \boldsymbol{u}^{(2)}\right)$ under the assumption that $P\left(\boldsymbol{v}^{(1)} \mid \boldsymbol{v}^{(2)}, \boldsymbol{u}^{(1)}, \boldsymbol{u}^{(2)}\right)=$ $P\left(\boldsymbol{v}^{(1)} \mid \boldsymbol{u}^{(1)}\right)$ and $P\left(\boldsymbol{v}^{(2)} \mid \boldsymbol{u}^{(1)}, \boldsymbol{u}^{(2)}\right)=P\left(\boldsymbol{v}^{(2)} \mid \boldsymbol{u}^{(2)}\right)$. This assumption is valid in the limit $\tau_{p} \rightarrow 0$. The particle velocity is well approximated by the flow velocity at the same point in this limit so that $P\left(\boldsymbol{v}^{(1)} \mid \boldsymbol{v}^{(2)}, \boldsymbol{u}^{(1)}, \boldsymbol{u}^{(2)}\right) \simeq \delta\left(\boldsymbol{v}^{(1)}-\boldsymbol{v}^{(2)}\right)$. Therefore it can be approximated by $P\left(\boldsymbol{v}^{(1)} \mid \boldsymbol{v}^{(2)}\right)$, which is also $\delta\left(\boldsymbol{v}^{(1)}-\boldsymbol{v}^{(2)}\right)$ in the limit. A similar argument applies to $P\left(\boldsymbol{v}^{(2)} \mid \boldsymbol{u}^{(1)}, \boldsymbol{u}^{(2)}\right)=P\left(\boldsymbol{v}^{(2)} \mid \boldsymbol{u}^{(2)}\right)$. In the other limit with $\tau_{p} \gg T_{L}$, we find the assumption for the conditional pdfs is also roughly valid. In this limit, due to the long memory, the particle velocity, $\boldsymbol{v}^{(1)}$ is not strongly correlated with the local flow velocity $\boldsymbol{u}^{(1)}$, nor with $\boldsymbol{u}^{(1)}$ or $\boldsymbol{u}^{(2)}$. Therefore $P\left(\boldsymbol{v}^{(1)} \mid \boldsymbol{v}^{(2)}, \boldsymbol{u}^{(1)}, \boldsymbol{u}^{(2)}\right)=P\left(\boldsymbol{v}^{(1)} \mid \boldsymbol{u}^{(1)}\right)$ could be a good approximation in the large particle limit. (The predicted relative velocity by this model could reproduce the two limits given in $\S 1$ ). However, for $\tau_{p}$ in the inertial range, all the four velocities are partially correlated. The assumption that neglects the "direct" 
correlation of the particle velocity at point (1) with the flow and particle velocities at point (2) would, to some degree, underestimate the particle velocity correlation.

From the 2-point joint pdf of particle and flow velocities, Zaichik et al. (2003) derived the joint pdf $P\left(\boldsymbol{v}^{(1)}, \boldsymbol{v}^{(2)}\right)$ and an analytical formula for the relative velocity. In the derivation, the Lagrangian correlation function is needed to calculate the velocity variance of each particle and the flow-particle correlation at each point, which fix $P\left(\boldsymbol{v}^{(1)} \mid \boldsymbol{u}^{(1)}\right)$ and $P\left(\boldsymbol{v}^{(2)} \mid \boldsymbol{u}^{(2)}\right)$ under the assumption of Gaussian statistics. With our eq. (2.16) for the Lagrangian correlation function, the model gives a linear scaling with $S t$ in the inertial range for the monodisperse case. This is in contrast to the $S t^{1 / 2}$ scaling in both their differential model and our model. Because the analytical model underestimates the velocity correlation, the predicted relative velocity for identical particles in the inertial range is much larger than in the latter two models, as well as than the results from simulations with low Reynolds numbers (Zaichik et al. 2003). Furthermore, the analytical model does not give a dip at $S t_{2} \simeq S t_{1}$ in the bidisperse case. This is again because the velocity correlation between similar particles is not accurately evaluated. The model does not account for the fact that similar size particles tend to have stronger correlation than different size ones.

\subsection{Derivich (2006)}

The model of Derivich (2006) starts with a similar approach as in the differential model of Zaichik et al. An equation for the joint pdf of both positions and velocities of the two particles is derived assuming Gaussian statistics for the flow velocity field. The equation is equivalent to that for the joint pdf of the particle separation and the relative velocity in Zaichik et al.'s model. Apparently, Derivich (2006) considered the solution of the joint pdf equation only in the zero separation limit $t$. Neglecting the spatial derivative terms in the joint pdf equation (i.e., assuming the spatial derivatives can be neglected in the zero separation limit), Derivich obtained a solution for the joint pdf (Gaussian) of the velocities of the two particles. The correlation of the particle velocities in the solution depends on the particle separation as a function of time (with initial separation set to be zero). In his calculations, the particle separation is taken to be a Gaussian variable. The time dependence of the separation variance is neglected, and apparently for the monodisperse case the variance is set to be a constant, corresponding to the particle distance at $\tau, \tau^{\prime}=-\tau_{p}$ in the ballistic separation behavior assumed in our model. We argue that this treatment with a constant particle separation variance is physically inadequate.

\subsection{Ayala et al. 2008}

Our formulation is very similar to that in Ayala et al. (2008). The model by Ayala et al. (2008) included the particle separation due to gravity for sedimenting droplets in turbulent flows, but neglected the particle separation by turbulent dispersion. In the absence of gravity, particles do not separate in their model, and the model enormously underestimates the relative velocity in the monodisperse case (where the separation plays a crucial role), as can be seen from their Fig. 11b. As explicitly pointed out by Ayala et al. (2008), their model was not designed for particles of similar sizes. In the presence of gravity, it is expected the accuracy of the model decreases with increasing turbulence intensity. This can be seen from their fig. 13 for the monodisperse case, where the predicted relative velocity agrees with the simulation results for the lower of the two turbulent intensities

$\dagger$ Zaichik et al. considered the whole range of the particle separations when solving their joint pdf equation and thus were able to examine the spatial clustering of particles that Derevich's model does not address. 
shown $\left(\bar{\epsilon}=100 \mathrm{~cm}^{2} / \mathrm{s}^{3}\right)$, while it is significantly smaller than the simulation results for the case with the higher intensity $\left(\bar{\epsilon}=400 \mathrm{~cm}^{2} / \mathrm{s}^{3}\right)$. Clearly, with larger turbulent intensity, the turbulent dispersion is faster and neglecting it would result in less reliable predictions. We also note that Ayala et al. adopted a bi-exponential form (similar to the form of our eq. (2.16) for the Lagarangian temporal correlation function) for the spatial correlation function of the flow velocity. The form corresponds to a linear velocity difference scaling and hence a -2 energy spectrum in the inertial range (for comparsion, see eq. (2.29) for the spatial correlation function adopted in our model), and is thus not consistent with the Kolmogorov spectrum observed in turbulent flows of high Reynolds numbers.

\section{Conclusions}

We have examined the relative velocity of inertial particles suspended in turbulent flows. A general formulation is established based on the calculation of the particle velocity structure function. Our general result for the particle structure function, eq. (2.12), has two terms, a generalized acceleration term, $\mathcal{A}_{i j}$ (eq. (2.13)), and a generalized shear term, $\mathcal{D}_{i j}$ (eq. (2.14)). The generalized shear term, $\mathcal{D}_{i j}$, corresponds to the contribution to the relative speed from particles' memory of the flow velocity difference in the past. We find that the backward-in-time dispersion of inertial particle pairs is needed to evaluate this term. The two terms reduce to the acceleration term and the shear term, respectively, in the S-T limit. Our formulation can thus be viewed as a generalization of Saffman and Turner's result for the limit of small particles to particles of any size.

We have shown that our model with a separation behavior similar to that found by recent simulations for the forward (in time) dispersion of inertial pairs (Bec et al. 2009a), i.e., a ballistic separation followed by a tracer-like behavior, gives quite good fits to the relative speed measured from simulations by Wang et al. (2000) (for the monodisperse case) and by Zhou et al. (2001) (for the bidisperse case).

For the monodisperse case, only $\mathcal{D}_{i j}$ contributes to the relative velocity. At large Reynolds numbers, a $S t^{1 / 2}$ scaling of the relative velocity in the inertial range is found for both the ballistic separation or the Richardson separation. Therefore, for the two-phase separation that well fits the simulation results, we have the same inertial-range scaling. This scaling is consistent with that from the differenical model by Zaichik and collaborators (Zaichik \& Alipchenkov 2003; Zaichik et al. 2003, 2006). Our model provides a clear physical picture for this scaling.

Our calculations for the bidisperse case show that $\mathcal{A}_{i j}$ dominates the contribution to the relative velocity between particles of very different sizes, while for similar particles the primary contribution is from $\mathcal{D}_{i j}$. In the relative velocity vs. $S t_{2}$ curves with fixed $S t_{1}$, dips are found around $S t_{2} \sim S t_{1}$, indicating stronger velocity correlation for similar size particles than for different size ones. Away from the dips, the relative velocity is essentially given by the contribution from $\mathcal{A}_{i j}$.

The main assumptions in our model are those for the trajectory correlation and trajectory structure tensors. The approximations for these tensors can be tested and improved by numerical simulations, and our work thus provides a motivation for direct studies of these correlations along the particle trajectories. A direct numerical study of the separation behavior of particles backward in time would also be of interest, because we have shown that it plays an important role in modeling the relative velocity between particles of similar sizes. With the help of future simulations, the assumptions in our model could be considerably refined. The model may also be extended to include gravity and other effects. The refined and extended model would provide a reliable prediction of the relative 
velocity between inertial particles and can be applied to many practical studies, such as raindrop formation in atmospheric clouds and collisions of dust grains in astrophysical environments.

LB acknowledges support from NASA grants 08-NAI5-0018 and NNX09AD10G.

\section{REFERENCES}

Abrahamson, J. 1975 Collision rates of small particles in a vigorously turbulent fluid. Chem. Eng. Sci. 30, 1371-1379

Ayala, O., Rosa, B. \& WAng, L.-P. 2008 Effects of turbulence on the geometric collision rate of sedimenting droplets. Part 2. Theory and parameterization. New J. Physics 10, 075016(1-40)

BATChelor G. K. 1950 The application of the similarity theory of turbulence to atmospheric diffusion. Quart. J. R. Meteorol. Soc. 76, 133-146

BAtchelor G. K. 1952 Diffusion in a field of homogeneous turbulence II. The relative motion of particles Math. Proc. Cam. Phil. Soc. 48,345-362

Batchelor, G. K., \& Townsend A. A. 1956 Turbulent diffusion. In Surveys in Mechanics (ed. Batchelor, G. K. \& Davies R. M.), pp. 352-399. Cambridge Univ. Press.

Bec, J., Celani, A.,Cencini, M., \& Musacchio, S. 2005 Clustering and collisions of heavy particles in random smooth flows. Phys. Fluids. 17, 073301

Bec, J., Biferale, L., Boffetta, G., Cencini, M., Musacchio, S., \& Toschi, F. 2006 Lyapunov exponents of heavy particles in turbulence. Phys. Fluids. 18, 091702

Bec, J., Cencini, M., \& Hillerbrand, R. 2007 Heavy particles in incompressible flows: The large Stokes number asymptotics. Physica D 226, 11-22

Bec, J., Biferale, L., Lanotte, A. S., Scagliarini, A., \& Toschi, F. 2009a Turbulent pair dispersion of inertial particles. arXiv: 0904.2314v1 [physics.fly-dyn]

Bec, J., Biferale, L., Cencini, M., Lanotte, A. S., \& Toschi, F. 2009b Caustics and Intermittency in Turbulent Suspensions of Heavy Particles. arXiv: 0905.1192v1 [physics.flydyn]

Berg, J., Luthi, B., Mann, J. \& Otт, S. 2006 Backwards and forwards relative dispersion in turbulent flow: An experimental investigation. Phys. Rev. E. 74, 016304(1-7)

Blum, J. \& Wurm, G. 2008 The Growth Mechanisms of Macroscopic Bodies in Protoplanetary Disks. Annu. Rev. Astron. Astrophys. 46, 21-56

Boffetta, G. \& Sokolov, I. M. 2002 Relative Dispersion in Fully Developed Turbulence: The Richardson's Law and Intermittency Corrections. Phys. Rev. Lett. 88, 094501(1-4)

Borgas, M. S. 1993 The Multifractal Lagrangian Nature of Turbulence. Philosophical Transactions: Physical Sciences and Engineering 342, 379-411

Corrsin, S. 1959 Progress Report on Some Turbulent Diffusion Research. Advances in Geophysics 6 ed Freinkel, F. N. and Sheppard P.A. (New York: Academic) 161-4

Cuzzi, J. N. \& Hogan R. C. 2003 Blowing in the windI. Velocities of chondrule-sized particles in a turbulent protoplanetary nebula. Icarus 164, 127-138

Derivich, I. V. 2006 Statistical modeling of particles relative motions in a turbulent gas flow. Int. J. Heat Mass Transfer 49, 4290-4304

Falkovich, G., Gawedzki, K., \& Vergassola, M. 2001 Particles and fields in fluid turbulence. Rev. Mod. Phys. 73, 913-75

Falkovich, G., Fouxon, A., \& Stepanov, M. G. 2002 Acceleration of rain initiation by cloud turbulence. Nature 419, 151-4

Falkovich, G. \& Pumir, A 2007 Sling Effect in Collisions of Water Droplets in Turbulent Clouds. J. Atmos. Sci. 64, 4497-505

Fouxon, I. \& Horvai, P. 2008 Separation of Heavy Particles in Turbulence. Phys. Rev. Lett. 100, 040601

Girimaji, S. S. \& Pope, S. B. 1990 Material-element deformation in isotropic turbulence. J. Fluid. Mech. 220, 427-458

Ishinara, T., Gotoh, T. \& Kaneda, Y. 2009 Study of High - Reynolds Number Isotropic Turbulence by Direct Numerical Simulation. Annu. Rev. Fluid Mech. 41, 165-180 
Kaneda, Y. \& Gotoh, T. 1991 Lagrangian velocity autocorrelation in isotropic turbulence. Phys. Fluids. A 3, 1924-1933

Kruis, F. E. \& Kusters, K. A. 1997 The Collision Rate of Particles in Turbulent Flow. Chem. Eng. Comm. 158, 201-230

Lundgren, T. S. 1981 Turbulent pair dispersion and scalar diffusion. J. Fluid Mech., 111, $27-57$

Markiewicz, W. J., Mizuno, H. \& Voelk, H. J. 1991 Turbulence induced relative velocity between two grains. Astronomy and Astrophysics 242, 286-289

Monin, A. S. \& Yaglom, A. M. 1975 Statistical Fluid Mechanics: Mechanics of Turbulence, vol. 2. MIT press.

Richardson, L. F.1926 Atmospheric Diffusion Shown on a Distance-Neighbour Graph. Proc. R. Soc. Lond. A 110, 709-737

Saffman, P. G. \& Turner, J. S. 1956 On the collision of drops in turbulent clouds. J. Fluid Mech. 1, 16-30

Salazar, J. P. L. C. \& Collins, L. R. 2009 Two-Particle Dispersion in Isotropic Turbulent Flows.Annu. Rev. Fluid Mech. 41, 405-432

SAWFORD, B. L. 1991 Reynolds number effects in Lagrangian stochastic models of turbulent dispersion. Phys. Fluids 3, 1577-1586

SAwford, B. L., Yeung, P. K. \& Borgas, M. S. 2005 Comparison of backwards and forwards relative dispersion in turbulence. Phys. Fluids 17, 095109 (1-9)

Sawford, B. L., Yeung, P. K. \& HackL, J. F. 2008 Reynolds number dependence of relative dispersion statistics in isotropic turbulence. Phys. Fluids 20, 065111 (1-13)

She, Z-S. \& Leveque, E. 1994 Universal scaling laws in fully developed turbulence. Phys. Rev. Lett. 72, 336-339

Shlien D. J.. \& Coorsin, S. 1974 A Measurement of Lagrangian Velocity auto-correlation in Approximately Isotropic Turbulence. J. Fluid Mech. 62, 255-271

Sundaram, S. \& Collins, L. R. 1997 Collision statistics in an isotropic particle-laden turbulent suspension. Part 1. Direct numerical simulations. J. Fluid Mech.335, 75-109

Volk, H. J., Jones, F. C., Morfill, G. E. \& Roeser, S. 1980 Collisions between grains in a turbulent gas. Astronomy and Astrophysics 85, 316-325

Voth, G. A., Satyanarayan, K. \& Bodenschatz, E. 1998 Lagrangian acceleration measurements at large Reynolds numbers. Phys. Fluids 10, 2268-2280

WANG, L.-P., WeXler, A. S. \& Zhou, Y. 2000 Statistical mechanical description and modelling of turbulent collision of inertial particles. J. Fluid Mech. 415, 117-153

Wilkinson, M. \& Mehlig, B. 2005 Caustics in turbulent aerosols. Europhys. Lett. 71, 186-192

Wilkinson, M. Mehlig, B., \& Bezuglyy, V. 2006 Caustic Activation of Rain Showers. Phys. Rev. Lett. 97, 048501

Weidenschilling, S. J. 1980 Dust to planetesimals: Settling and coagulation in the solar nebula. Icarus 44, 172-189

Williams, J. J. E. \& Crane, R. I. 1983 Particle collision rate in turbulent flow. Int. J. Multiphase Flow 9, 421-435

Yeung, P. K. \& Pope, S. B 1989 Lagrangian statistics from direct numerical simulations of isotropic turbulence. J. Fluid. Mech. 207, 531-586

Yeung, P. K., Pope, S. B. \& SAwford, B. L. 2006a Reynolds number dependence of Lagrangian statistics in large numerical simulations of isotropic turbulence. J. Turbulence $\mathbf{7}$, $58(1-12)$

Yeung, P. K., Pope, S. B., Lamorgese, A. G. \& Donzis, D. A. 2006b Acceleration and dissipation statistics of numerically simulated isotropic turbulence. Phys. Fluids 18, 065103 $(1-14)$

YUU, S. 1984 Collision rate of small particles in a homogeneous and isotropic turbulence. AIChE J. 30, 802-807

Zaichik, L. I. \& Alipchenkov, V. M. 2003 Pair dispersion and preferential concentration of particles in isotropic turbulence. Phys. Fluids 15, 1776-1787

Zaichik, L. I., Simonin, O. \& Alipchenkov, V. M. 2003 Two statistical models for predicting collision rates of inertial particles in homogeneous isotropic turbulence. Phys. Fluids. 15, 2995-3005 
Zaichik, L. I., Simonin, O. \& Alipchenkov, V. M. 2006 Collision rates of bidisperse inertial particles in isotropic turbulence. Phys. Fluids 18, 035110 (1-13)

Zhou, Y., Wexler, A. S. \& WANG, L.-P. 2001 Modelling turbulent collision of bidisperse inertial particles. J. Fluid Mech. 433, 77-104 